\title{
Selecting a differential equation cell cycle model for simulating leukemia treatment
}

María Fuentes-Garí, ${ }^{\dagger}$ Ruth Misener, ${ }^{\ddagger}$ Michael C. Georgiadis, $₫$ Margaritis Kostoglou, ${ }^{\S}$ Nicki Panoskaltsis, $\|$ Athanasios Mantalaris, ${ }^{*, \dagger}$ and Efstratios N. Pistikopoulos $*, \perp$

${ }^{\dagger}$ Department of Chemical Engineering, Imperial College London, SW7 2AZ, London, UK, ${ }^{\ddagger}$ Department of Computing, Imperial College London, SW7 2AZ, London, UK,

"Department of Chemical Engineering, Aristotle University of Thessaloniki, Thessaloniki, Greece, ${ }^{\S}$ Department of Chemistry, Aristotle University of Thessaloniki, Thessaloniki, Greece, "Centre for Haematology, Imperial College London, Northwick Park Campus, HA1 $3 L Y$, London, UK, and ${ }^{\perp}$ Artie McFerrin Department of Chemical Engineering, Texas A $8 M$ University, College Station, 77843 TX USA

E-mail: *a.mantalaris@imperial.ac.uk; *stratos@tamu.edu Phone: +1-979-458-02590. Fax: +1-979-845-0593 


\begin{abstract}
This manuscript studies three differential equation models of the leukemia cell cycle: a population balance model (PBM) using intracellular protein expression levels as state variables representing phase progress; a delay differential equation model (DDE) with temporal phase durations as delays; and an ordinary differential equation model (ODE) of inter-phase progression. In each type of model, global sensitivity analysis determines the most significant parameters while parameter estimation fits experimental data. In order to compare models based on the output of their structural properties, an expected behavior was defined, and each model was coupled to a pharmacokinetic/pharmacodynamic model of chemotherapy delivery. Results suggest that the particular cell cycle model chosen highly affects the simulated treatment outcome, given the same steady state kinetic parameters and drug dosage/scheduling. The manuscript shows how cell cycle models should be selected according to the complexity, sensitivity and parameter availability of the application envisioned.
\end{abstract}




\section{Introduction}

Acute Myeloid Leukemia (AML) is a type of blood cancer characterized by a rapid increase in immature blood cells with highly proliferative features. ${ }^{1}$ Chemotherapy treatments rely on cell duplication cycle phase-specific (CCS) agents, which are specific to cells going through one of the four phases of the cell duplication cycle. ${ }^{2}$ The cell cycle is the four phase (G1-SG2-M) biological process used by cells to replicate their genetic material and give birth to new cells. ${ }^{3}$ The cell cycle is highly regulated by the timed expression of proteins (cyclins) triggering cell cycle events such as the start of DNA replication (cyclin E, peaking at the end of G1) or the commencement of mitosis (when the cell physically divides into two daughter cells - cyclin B, peaking at the end of G2). ${ }^{4}$ Mathematical models of the cell cycle have been widely developed both at the mechanistic and descriptive levels. ${ }^{5}$ Mechanistic models typically represent protein networks or other biological signals ("high amounts of transition protein cyclin E will result in likely transition to S phase") in an effort to explain the underlying causes for cell growth. ${ }^{6,7}$ In contrast, descriptive models represent observable cause-effect phenomena ("one cell gives birth to another cell after $24 \mathrm{~h}$ ") as an explanation of the overall system behavior. ${ }^{8-11}$ Some hybrid mechanistic/descriptive approaches have arisen, benefiting from the advantages of both (the in-depth insights in mechanistic models, and the fast computation of key variables in descriptive models). Due to the cell cycle specificity of most chemotherapeutic drugs, mathematical models are increasingly used to study and simulate cellular kinetics in response to cancer treatment. ${ }^{12,13}$ However, choosing appropriate models is critical for accurate clinical predictions. ${ }^{14}$ More specifically, models should be selected according to expected dynamic behavior and parameter identifiability. ${ }^{15,16}$

Current treatment protocols efficacy is hampered by a lack of patient-specific information related to drug action and cell cycle kinetics. In AML, bone marrow samples from patients receiving chemotherapy cannot be collected due to their medical condition and to weakening treatment effects. Therefore, modeling patient response in silico could provide valuable insights as a tool for clinicians. ${ }^{17,18}$ To this end, Pefani et al. ${ }^{19,20}$ developed a complete phar- 
macokinetic/pharmacodynamic (PK/PD) model of patient response; the model considered infusion, transport and degradation of chemotherapeutic drugs inside the body, as well as drug effects at the point of action (bone marrow). The leukemic cell cycle was represented as a set of 3 ODEs, one per compartment (cell number totals per phase: G1, S and G2/M). Global sensitivity analysis revealed that the cell cycle phase durations $\left(\tau_{G}, \tau_{S}\right.$ and $\tau_{M}$ in hours) were the most significant parameters for treatment outcomes. However, ODEs do not describe intra-phase coordinates but an averaged phase behavior. Since cell cycle phase ODEs (CCP-ODEs) provide only a simplified representation of cell cycle progression, we hypothesize that the cell cycle model itself, in addition to its parameters, would have an impact on simulated chemotherapy response. More complex types of models such as population balance models (PBM) which are distributed in a second progression-related variable, or delay differential equation models (DDE) which account for the phase-induced time delay, could be more suited for the purpose. The cell cycle is typically an oscillating system; its equilibrium lies at the steady state cell cycle distributions and log-growth. However, when taken out of the equilibrium, cell fractions undergo a transient state that is characterized by the oscillatory properties of the specific model chosen. ${ }^{21}$ Under chemotherapy treatment, oscillations play a key role as they determine how much room there is for chemotherapy action (favorable times: target phase highs; unfavorable times: target phase lows). ${ }^{22}$

In this paper, we present three cell cycle phase models whose parameters can be measured experimentally. Two of them have been previously proposed: the cell cycle phase PBM (CCP-PBM) developed by Fuentes-Garí et al. ${ }^{23}$ and García Münzer et al.; ${ }^{24,25}$ and the Pefani et al. ${ }^{19}$ CCP-ODE model. A new cell cycle phase DDE (CCP-DDE) model featuring phase-specific time delays is presented here as an example of a DDE model with measurable parameters. DDE cell cycle models have been widely studied by Colijn and Mackey, ${ }^{26,27}$ considering proliferating and resting cell cycle states, but the parameters required are difficult to obtain experimentally. All three models (CCP-ODE, CCP-PBM, CCP-DDE) are compared in: (i) their ability to fit chemotherapy-free experimental data; ${ }^{23}$ (ii) chemother- 
apy outcomes when embedded in the pharmacokinetic/pharmacodynamic (PK/PD) model of Pefani et al.. ${ }^{19}$

\section{Model definitions}

\section{Development of three differential phase kinetics cell cycle models}

Models are presented with increasing levels of complexity (Figure 1, bottom to top). Phase

distributions (percents) are important as they account for oscillatory behavior, in contrast with phase numbers which increase over time. These distributions can be experimentally measured by aggregating data from single cell DNA content measurement techniques into averaged population data.

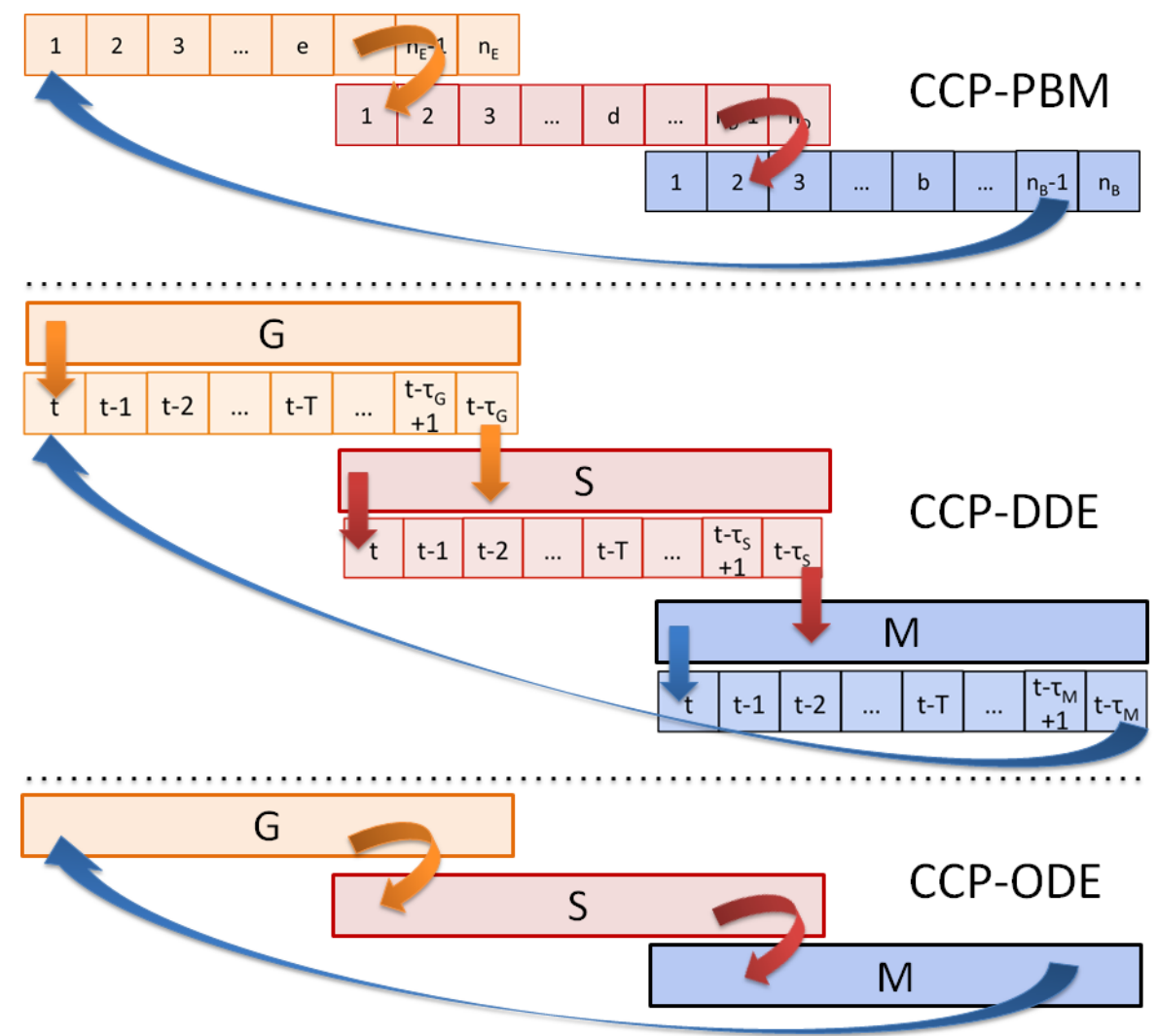

Figure 1: Model structure in CCP-PBM, CCP-DDE, CCP-ODE with discretization intervals for CCP-PBM, history vectors and lumped phase compartments for CCP-DDE and lumped phase compartments only for CCP-ODE. 
An initial approach is to model cell cycle phases with ODEs, with the parameters needed being the transition rates and the initial cell populations for each phase (refer to Table S2 in Supporting Information for variable and parameter definitions). Pefani et al. ${ }^{19}$ give an example with three compartments (G0/G1, S and G2/M):

$$
\begin{aligned}
\frac{d G}{d t} & =2 \cdot M / \tau_{M}-G / \tau_{G} \quad \text { or } \\
\frac{d G_{\%}}{d t} & =2 \cdot M_{\%} / \tau_{M}-G_{\%} \cdot\left(1 / \tau_{G}+\frac{1}{T} \frac{d T}{d t}\right) \\
\frac{d S}{d t} & =G / \tau_{G}-S / \tau_{S} \quad \text { or } \\
\frac{d S_{\%}}{d t} & =G \% / \tau_{G}-S_{\%} \cdot\left(1 / \tau_{S}+\frac{1}{T} \frac{d T}{d t}\right) \\
\frac{d M}{d t} & =S / \tau_{S}-M / \tau_{M} \quad \text { or } \\
\frac{d M_{\%}}{d t} & =S_{\%} / \tau_{S}-M_{\%} \cdot\left(1 / \tau_{M}+\frac{1}{T} \frac{d T}{d t}\right) \\
T & =G+S+M
\end{aligned}
$$

where $G, S$ and $M$ are the cell numbers in each of G0/G1, S and G2/M phases (in \# cells), $T$ is the total number of cells (in \# cells), $G_{\%}, S_{\%}$ and $M_{\%}$ are the phase fractions of total cells (in \%) and $\tau_{G}, \tau_{S}$ and $\tau_{M}$ are the phase durations (in hours) of the G0/G1, S and G2/M phases respectively. An alternative model accounts for the temporal discrepancy between cells entering and exiting phases. For this, we developed a new DDE model by introducing 
a time delay equal to phase duration:

$$
\begin{aligned}
\frac{d G(t)}{d t} & =2 \cdot M\left(t-\tau_{M}\right) / \tau_{M}-G(t) / \tau_{G} \text { or } \\
\frac{d G_{\%}(t)}{d t} & =2 \cdot M_{\%}\left(t-\tau_{M}\right) / \tau_{M}-G_{\%}(t) \cdot\left(1 / \tau_{G}+\frac{1}{T(t)} \frac{d T(t)}{d t}\right) \\
\frac{d S(t)}{d t} & =G\left(t-\tau_{G}\right) / \tau_{G}-S(t) / \tau_{S} \quad \text { or } \\
\frac{d S_{\%}(t)}{d t} & =G_{\%}\left(t-\tau_{G}\right) / \tau_{G}-S_{\%}(t) \cdot\left(1 / \tau_{S}+\frac{1}{T(t)} \frac{d T(t)}{d t}\right) \\
\frac{d M(t)}{d t} & =S\left(t-\tau_{S}\right) / \tau_{S}-M(t) / \tau_{M} \text { or } \\
\frac{d M_{\%}(t)}{d t} & =S_{\%}\left(t-\tau_{S}\right) / \tau_{S}-M_{\%}(t) \cdot\left(1 / \tau_{M}+\frac{1}{T(t)} \frac{d T(t)}{d t}\right) \\
T(t) & =G(t)+S(t)+M(t)
\end{aligned}
$$

where all variables and parameters are defined as in CCP-ODE and $G\left(t-\tau_{G}\right), S\left(t-\tau_{S}\right)$, $M\left(t-\tau_{M}\right)$ (in \# cells) represent the phase cell number at times $t-\tau_{G}, t-\tau_{S}$ and $t-$ $\tau_{M}$ respectively (refer to Table S3 in Supporting Information for variable and parameter definitions). Note that the system is not completely closed $\left(G(t) / \tau_{G}\right.$ cells exiting the $\mathrm{G}$ phase versus $G\left(t-\tau_{G}\right) / \tau_{G}$ cells entering the $\mathrm{S}$ phase), however this results in very small $(<3 \%)$ variations in total cell number which are transient (total cell number is conserved at the steady state, see Section F in Supporting Information). An important advantage of DDEs is that they add a phase coordinate dimension to the system (i.e., cell populations are not eligible to exit a phase as soon as they enter), so any disturbances in time will be well captured. Furthermore, CCP-DDE does not require any additional parameters and maintains a fast computational execution (see Parts A and B in the Supporting Information for DDE discretization details and initialization equations).

Because phase to phase transition in CCP-DDE systems is based on total phase numbers at earlier times, not intra-phase cell numbers, properties related to phase progression cannot be deduced. In order to account for properties varying within each phase, distributed systems such as PBMs must be used. Previously developed PBMs rely on state variables that are not always measurable and/or do not maintain fidelity to the underlying biology. ${ }^{8}$ To make 
this biologically relevant, those properties should be experimentally measurable. In FuentesGarí et al., ${ }^{23}$ we presented a CCP-PBM of the cell cycle with cyclins E and B $\left(C_{E}\right.$ and $C_{B}$, proteins related to phase progression; normalized \% expression) as state variables for G0/G1 and G2/M, where their concentration actively increases throughout the phase, and DNA content (in DNA units) as state variable for S phase (where DNA replication occurs):

$$
\begin{aligned}
& \frac{\partial G\left(\mathrm{C}_{E}, t\right)}{\partial t}+\frac{\partial\left(G\left(\mathrm{C}_{E}, t\right) \cdot \frac{d \mathrm{C}_{E}}{d t}\right)}{\partial \mathrm{C}_{E}}=-r_{G \rightarrow S}\left(\mathrm{C}_{E}\right) \cdot G\left(\mathrm{C}_{E}, t\right) \\
& \frac{\partial S(\mathrm{DNA}, t)}{\partial t}+\frac{\partial\left(S(\mathrm{DNA}, t) \cdot \frac{d \mathrm{DNA}}{d t}\right)}{\partial \mathrm{DNA}}=0 \\
& \frac{\partial M\left(\mathrm{C}_{B}, t\right)}{\partial t}+\frac{\partial\left(M\left(\mathrm{C}_{B}, t\right) \cdot \frac{d \mathrm{C}_{B}}{d t}\right)}{\partial \mathrm{C}_{B}}=-r_{M \rightarrow G}\left(\mathrm{C}_{B}\right) \cdot M\left(\mathrm{C}_{B}, t\right)
\end{aligned}
$$

and the boundary conditions are written:

$$
\begin{gathered}
r_{G} \cdot G\left(\mathrm{C}_{E}=\mathrm{C}_{E, \text { min }}, t\right)=2 \cdot \int_{\mathrm{C}_{B, \min }}^{\mathrm{C}_{B, \max }} r_{M \rightarrow G}\left(\mathrm{C}_{B}\right) \cdot M\left(\mathrm{C}_{B}, t\right) d \mathrm{C}_{B} \\
r_{S} \cdot S(\mathrm{DNA}=1, t)=\int_{\mathrm{C}_{E, \text { min }}}^{\mathrm{C}_{E, \text { max }}} r_{G \rightarrow S}\left(\mathrm{C}_{E}\right) \cdot G\left(\mathrm{C}_{E}, t\right) d \mathrm{C}_{E} \\
r_{M} \cdot M\left(\mathrm{C}_{B}=\mathrm{C}_{B, \min }, t\right)=r_{S} \cdot S(\mathrm{DNA}=2, t)
\end{gathered}
$$

Growth rates $\left(r_{G}=d C_{E} / d t, r_{S}=d D N A / d t\right.$ and $\left.r_{M}=d C_{B} / d t ; \mathrm{h}^{-1}\right)$ correlate phase progression to its state variable and are constant (see definition in Appendix C); transition rates $\left(r_{G \rightarrow S}\left(C_{E}\right)\right.$ and $\left.r_{M \rightarrow G}\left(C_{B}\right) ; \mathrm{h}^{-1}\right)$ account for the transition probability according to the state variable level (see Part C and Table S4 in the Supporting Information for details). The model was discretized in cyclin $\mathrm{E}$ (resulting in $n_{E}$ bins with indices $e \in\left\{1, \ldots, n_{E}\right\}$ ), in cyclin $\mathrm{B}$ (resulting in $n_{B}$ bins with indices $b \in\left\{1, \ldots, n_{B}\right\}$ ) and in DNA (resulting in $n_{D}$ bins with indices $\left.d \in\left\{1, \ldots, n_{D}\right\}\right)$ and validated for 3 leukemia cell lines (K-562, MEC-1, 
MOLT-4) experimentally:

$$
\begin{aligned}
\frac{d G_{e}}{d t}= & (1-\delta(e-1)) \cdot G_{e-1} \cdot r_{G} \cdot \nu_{E}-G_{e} \cdot \nu_{E} \cdot\left(r_{G}+r_{G \rightarrow S, e}\right) \\
& +\delta(e-1) \cdot 2 \cdot \sum_{b=1}^{n_{B}} M_{b} \cdot r_{M \rightarrow G, b} \cdot \nu_{B} \\
\frac{d G_{e, G \%}}{d t}= & (1-\delta(e-1)) \cdot G_{e-1, G \%} \cdot r_{G} \cdot \nu_{E}-G_{e, G \%} \cdot\left(r_{G} \cdot \nu_{E}+r_{G \rightarrow S, e} \cdot \nu_{E}+\frac{d G}{d t} \cdot \frac{1}{G}\right) \\
& +\delta(e-1) \cdot \frac{2}{G \%} \cdot \sum_{b=1}^{n_{B}} M_{b, T \%} \cdot r_{M \rightarrow G, b} \cdot \nu_{B} \quad \forall e \in\left\{1, \ldots, n_{E}\right\} \\
\frac{d S_{d}}{d t}= & (1-\delta(d-1)) \cdot S_{d-1} \cdot r_{S} \cdot \nu_{D}-S_{d} \cdot r_{S} \cdot \nu_{D}+\delta(d-1) \cdot \sum_{e=1}^{n_{E}} G_{e} \cdot r_{G \rightarrow S, e} \cdot \nu_{E} \\
\frac{d S_{d, S \%}}{d t}= & (1-\delta(d-1)) \cdot S_{d-1, S \%} \cdot r_{S} \cdot \nu_{D}-S_{d, S \%} \cdot\left(r_{S} \cdot \nu_{D}+\frac{d S}{d t} \cdot \frac{1}{S}\right) \\
& +\delta(d-1) \cdot \frac{1}{S \%} \cdot \sum_{e=1}^{n_{E}} G_{e, T \%} \cdot r_{G \rightarrow S, e} \cdot \nu_{E} \quad \forall d \in\left\{1, \ldots, n_{D}\right\} \\
\frac{d M_{b}}{d t}= & (1-\delta(b-1)) \cdot M_{b-1} \cdot r_{M} \cdot \nu_{B}-M_{b} \cdot \nu_{B} \cdot\left(r_{M}+r_{M \rightarrow G, b}\right)+\delta(b-1) \cdot S_{n_{D}} \cdot r_{S} \cdot \nu_{D} \\
\frac{d M_{b, M \%}}{d t}= & (1-\delta(b-1)) \cdot M_{b-1, M \%} \cdot r_{M} \cdot \nu_{B}-M_{b, M \%} \cdot\left(r_{M} \cdot \nu_{B}+r_{M \rightarrow G, b} \cdot \nu_{B}+\frac{d M}{d t} \cdot \frac{1}{M}\right) \\
& +\delta(b-1) \cdot \frac{1}{M_{\%}} \cdot S_{n_{D}, T \%} \cdot r_{S} \cdot \nu_{D} \quad \forall b \in\left\{1, \ldots, n_{B}\right\} \\
& \quad G=\sum_{e=1}^{n_{E}} G_{e}, S=\sum_{d=1}^{n_{D}} S_{d}, \text { and } M=\sum_{b=1}^{n_{B}} M_{b} ; \\
\text { with: } & \quad G_{e, G \%}=G_{e} / G, S_{d, S \%}=S_{d} / S, \text { and } M_{b, M \%}=M_{b} / M \\
\text { and } &
\end{aligned}
$$

And the total being:

$$
T(t)=\sum_{e=1}^{n_{E}} G_{e}+\sum_{d=1}^{n_{D}} S_{d}+\sum_{b=1}^{n_{B}} M_{b}
$$

with: $\quad G_{e, T \%}=G_{e} / T, S_{d, T \%}=S_{d} / T$, and $M_{b, T \%}=M_{b} / T$

(CCP-PBM)

The parameters $\nu_{E}, \nu_{D}$ and $\nu_{B}$ represent the conversion factor from bins to cyclin expression and are a result of the discretization method, in G0/G1, S and G2/M phases. The terms $r_{G \rightarrow S, e}$ and $r_{M \rightarrow G, b}\left(\mathrm{~h}^{-1}\right)$ account for the discretized transition rates in each of the G0/G1 ( $e$ bins) or $\mathrm{G} 2 / \mathrm{M}(b$ bins) phases. $\delta(x)$ takes the values 1 for $x=0$ and 0 for any other value of $x$. A summary of model parameters and how to obtain them is shown in Table 1. 
Table 1: Model parameters and experimental methods needed to obtain them (the model source is clearly stated, otherwise all models use that specific parameter).

\begin{tabular}{|c|c|c|c|}
\hline Parameter & $\begin{array}{c}\text { Lab } \\
\text { protocol }\end{array}$ & $\begin{array}{l}\text { Lab } \\
\text { time }\end{array}$ & $\begin{array}{c}\text { Data } \\
\text { processing }\end{array}$ \\
\hline$\tau_{G}, \tau_{S}, \tau_{M}$ & $\begin{array}{l}\text { Cell cycle chase: } \\
\text { In vitro culture }(>24 \mathrm{~h}) \\
\text { Frequent sampling }\end{array}$ & $40-60 \mathrm{~h}$ & $10-15 \mathrm{~h}$ \\
\hline $\begin{array}{c}G_{S S, \%}, S_{S S, \%}, M_{S S, \%} \\
G_{0, \%}, S_{0, \%}, M_{0, \%}\end{array}$ & $\begin{array}{l}\text { No culturing needed } \\
\text { Initial time point only } \\
\text { Fast sample preparation }\end{array}$ & $2-3 \mathrm{~h}$ & $10 \mathrm{~min}$ \\
\hline $\begin{array}{l}\mu(\mathrm{CCP}-\mathrm{ODE}) \\
\gamma(\mathrm{CCP}-\mathrm{DDE}) \\
\alpha(\mathrm{CCP}-\mathrm{PBM})\end{array}$ & $\begin{array}{c}2 \text { samples needed } \\
\text { either in vitro culture } \\
\text { or in vivo blood sample }\end{array}$ & $5-10 \mathrm{~h}$ & $10-15 \mathrm{~min}$ \\
\hline $\begin{array}{c}\text { CCP-PBM: } \sigma_{B}, \sigma_{E}, \\
\mathrm{C}_{B, \min }, \mathrm{C}_{E, \min }, \\
\mathrm{C}_{B, \max }, \mathrm{C}_{E, \max }\end{array}$ & $\begin{array}{l}1 \text { sample needed } \\
\text { DNA, cyclin E\&B labelling } \\
\text { segregate } 2 \text { subpopulations }\end{array}$ & $10-15 \mathrm{~h}$ & $1-2 \mathrm{~h}$ \\
\hline
\end{tabular}

\section{Solution of the steady state problem}

The system's steady state in population balance models is frequently studied for nucleation and crystal growth processes. ${ }^{28}$ In the cell duplication context, cell cycle phases may reach steady state with respect to the percentage of cells in each phase $\left(G_{\%}, S_{\%}, M_{\%}\right)$ but not in the phases themselves $(G, S, M)$ as the absolute count will increase over time as a result of doubling. Cell cycle models usually oscillate initially when they are taken out of their equilibrium cell cycle distribution (transient state) and progressively reach steady state as they approach phase equilibrium. If these models are initialized at equilibrium, they should not oscillate. The equilibrium cell cycle phase distributions are determined by setting their derivative to zero (see Part D in the Supporting Information for an extended derivation).

For CCP-ODEs, the equilibrium cell cycle phase distributions are given by the real root of $\mathrm{Eq}(30)$ in the SI for $G_{\%, S S}$ and by $\mathrm{Eq}(27)$ and (28) in the SI for $M_{\%, S S}$ and $S_{\%, S S}$ respectively. The log-growth coefficient is calculated as:

$$
\frac{d T}{d t} \frac{1}{T}=\frac{1}{\tau_{M}} \cdot M_{\%, S S}=\mu
$$


Similarly, Eq (43)-(45) in the SI represent the steady state cell cycle distribution for CCPDDEs, with log-growth coefficient:

$\frac{d T}{d t} \cdot \frac{1}{T}=\frac{1}{\tau_{M}} \cdot M_{S S, \%} \cdot\left(2 \cdot e^{-\gamma \cdot \tau_{M}}-1\right)+\frac{1}{\tau_{G}} \cdot G_{S S, \%} \cdot\left(e^{-\gamma \cdot \tau_{G}}-1\right)+\frac{1}{\tau_{S}} \cdot S_{S S, \%} \cdot\left(e^{-\gamma \cdot \tau_{S}}-1\right)=\gamma$

Finally, the steady state cell cycle distribution (intra-phase as well as phase totals if summed) for CCP-PBM is given by Eq (76)-(78) in SI, with the log-growth coefficient calculated as:

$$
\frac{d T}{d t} \cdot \frac{1}{T}=\sum_{b=1}^{n_{B}} M_{b, S S, T \%} \cdot r_{M \rightarrow G, b} \cdot \nu_{B}=\alpha
$$

\section{Embedding CC models into a previously developed PK/PD model}

Pefani et al. ${ }^{19}$ developed a PK/PD model simulating patient response to chemotherapy delivery with two common drugs, cytarabine (Ara-C) and daunorubicin (DNR). Ara-C acts by in terfering with DNA duplication (in $\mathrm{S}$ phase only). ${ }^{29}$ DNR attacks $\mathrm{S}$ phase cells by DNA intercalation but also G1 cells by inhibition of macromolecular biosynthesis. ${ }^{30}$ The treatment prescribed by the physician is used as an input variable consisting of drug dose and administration route. Parameters adjustable on a case-by-case basis included height and weight, age and cell cycle times (Figure 2). Pharmacokinetics (PK) considers the transport and transformation the drug undergoes once it reaches the blood streams, and in turn the relevant organs which absorb it at different rates. ${ }^{31}$ Mass balances are performed in each of these organs, giving the drug concentration profiles, which are the main input for pharmacodynamics (PD). In the PD model, the drug effect on cells is computed according to cell cycle kinetics. Since chemotherapy acts only during specific cell cycle phases, detailed cell cycle equations modeling the point of drug action are needed (as confirmed by their global sensitivity analysis).

The drug effect term is applied differently in each model. The effects occur only in the $G$ and $S$ compartments and are accounted for through $k_{d, G}$ and $k_{d, S}$ death rates, respectively. 


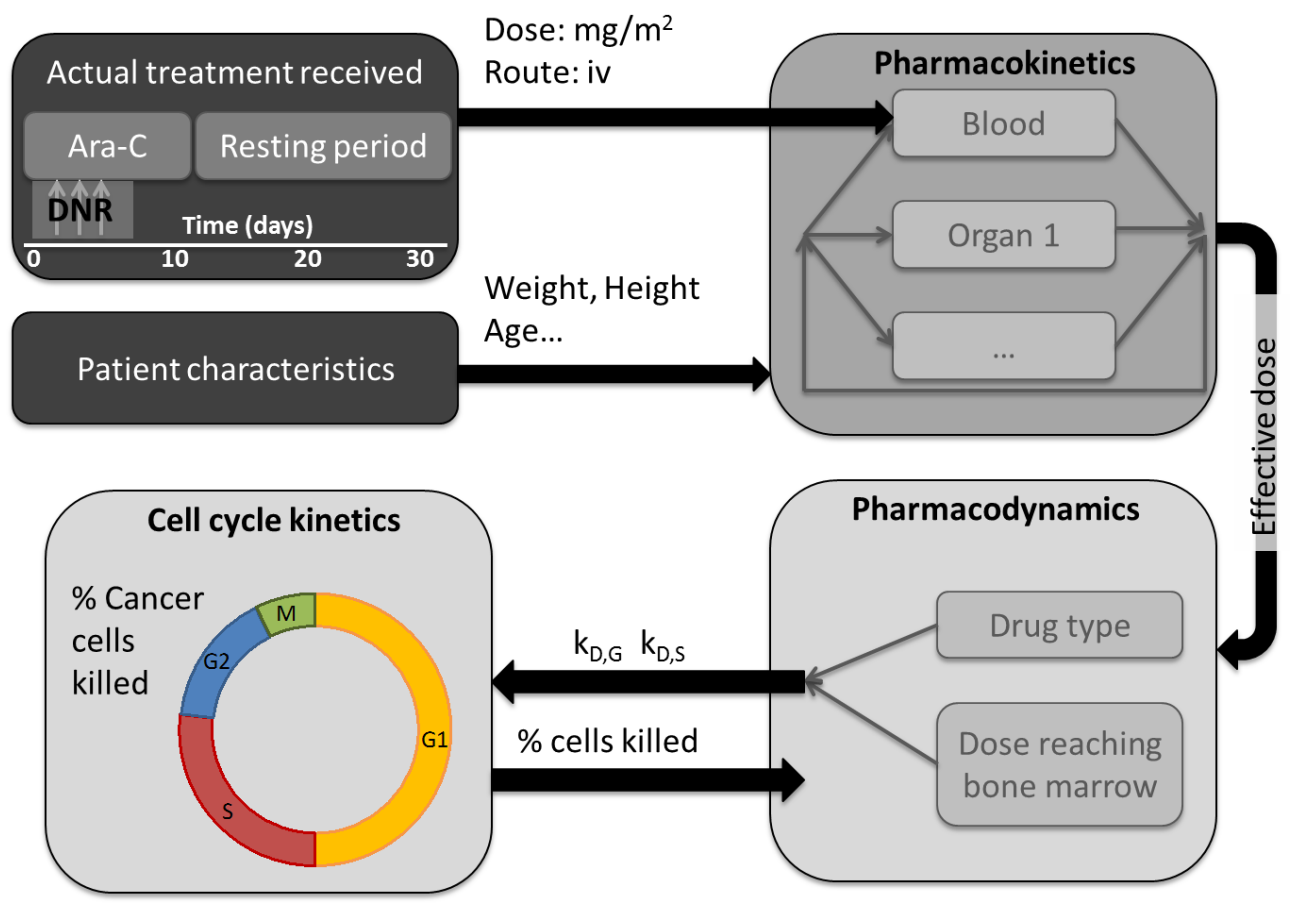

Figure 2: Schematic of the interactions between PK, PD and cell cycle components of the Pefani et al. model, together with input of patient data.

In the original CCP-ODE model, they are included directly into each equation as:

$$
\begin{aligned}
\frac{d G}{d t} & =2 \cdot M / \tau_{M}-G / \tau_{G}-k_{D, G} \cdot G \\
\frac{d S}{d t} & =G / \tau_{G}-S / \tau_{S} \quad-k_{D, S} \cdot S \\
\frac{d M}{d t} & =S / \tau_{S}-M / \tau_{M}
\end{aligned}
$$

When moving to CCP-DDE, the temporal delay has to be considered: cells in those phases 
are subject to chemotherapy for as long as the delay lasts.

$$
\begin{aligned}
\frac{d G(t)}{d t} & =2 \cdot M\left(t-\tau_{M}\right) / \tau_{M}-G(t) / \tau_{G} \quad-k_{D, G}(t) \cdot G(t) \\
\frac{d S(t)}{d t} & =G^{*}\left(\tau_{G}\right) / \tau_{G}-S(t) / \tau_{S} \quad-k_{D, S}(t) \cdot S(t) \\
\frac{d M(t)}{d t} & =S^{*}\left(\tau_{S}\right) / \tau_{S}-M(t) / \tau_{M} \quad \text { where: } \\
\frac{d G^{*}\left(t_{G}^{*}\right)}{d t_{G}^{*}} & =-k_{d, G} \cdot G^{*}\left(t_{G}^{*}\right) \quad \text { with } \quad G^{*}\left(t_{G}^{*}=0\right)=G\left(t-\tau_{G}\right) \\
& \text { or: } \quad G^{*}\left(\tau_{G}\right)=G\left(t-\tau_{G}\right) \cdot e^{-k_{d, G} \cdot \tau_{G}} \\
\frac{d S^{*}\left(t_{S}^{*}\right)}{d t_{S}^{*}} & =-k_{d, S} \cdot S^{*}\left(t_{S}^{*}\right) \quad \text { with } \quad S^{*}\left(t_{S}^{*}=0\right)=S\left(t-\tau_{S}\right) \\
& \text { or: } \quad S^{*}\left(\tau_{S}\right)=S\left(t-\tau_{S}\right) \cdot e^{-k_{d, S} \cdot \tau_{S}}
\end{aligned}
$$

Finally, the drug is applied similarly to CCP-PBM, in a homogeneous manner throughout the relevant phases:

$$
\begin{aligned}
\frac{d G_{e}}{d t}= & (1-\delta(e-1)) \cdot G_{e-1} \cdot r_{G} \cdot \nu_{E}-G_{e} \cdot\left(r_{G} \cdot \nu_{E}+r_{G \rightarrow S, e} \cdot \nu_{E}+k_{d, G}\right) \\
& +2 \delta(e-1) \cdot \sum_{b=1}^{n_{B}} M_{b} \cdot r_{M \rightarrow G, b} \cdot \nu_{B} \quad \forall e \in\left\{1, \ldots, n_{E}\right\} \\
\frac{d S_{d}}{d t}= & (1-\delta(d-1)) \cdot S_{d-1} \cdot r_{S} \cdot \nu_{D}-S_{d} \cdot\left(r_{S} \cdot \nu_{D}+k_{d, S}\right)+\delta(d-1) \cdot \sum_{e=1}^{n_{E}} G_{e} \cdot r_{G \rightarrow S, e} \cdot \nu_{E} \\
& \forall d \in\left\{1, \ldots, n_{D}\right\} \\
\frac{d M_{b}}{d t}= & (1-\delta(b-1)) \cdot M_{b-1} \cdot r_{M} \cdot \nu_{B}-M_{b} \cdot \nu_{B} \cdot\left(r_{M}+r_{M \rightarrow G, b}\right)+\delta(b-1) \cdot S_{n_{D}} \cdot r_{S} \cdot \nu_{D} \\
& \forall b \in\left\{1, \ldots, n_{B}\right\}
\end{aligned}
$$

(CCP-PBM-PD)

Note that the percentage of cells killed at a given time point is the same in all three models, however CCP-DDE-PD and CCP-PBM-PD incorporate drug effects (due to phase distribution in either time or space) in a time-cumulative manner while CCP-ODE-PD only account for instantaneous effects of that particular dose. 


\section{Results and discussion}

The objective of this paper is to answer the question: are the models under study suitable for the application envisioned? To address this question, we will first deal with the case where no treatment is applied (exponential growth conditions) and in the second part the chemotherapy will be incorporated. Several aspects are to be considered under both cases.

\section{Analysis under exponential growth conditions}

\section{Satisfying short-term kinetics}

Defining the transient state requires identifying the significant parameters for each model short-term (for the first cell cycle) and finding their values through parameter estimation with experimental data.

What parameters are needed short-term? Global sensitivity analysis (GSA) was performed for all three models by varying the parameters they have in common (phase durations) $40 \%$ around their nominal (experimental) values simultaneously following Sobol's method (Section E in SI), and observing the effect on the model output for phase fractions. For each model, the number of intervals (model evaluations) required to obtain accurate estimates is found to be different: 5,000 intervals for CCP-ODE, 2,000 intervals in CCP-DDE and 20,000 intervals for CCP-PBM. The sensitivity indexes were compared for the three phases (Figure 3 ) over one cell cycle $(22 \mathrm{~h})$ at $0 \mathrm{~h}, 1 \mathrm{~h}$ and $5 \mathrm{~h}$ intervals subsequently. It was confirmed that phase times were significant, especially in their own (cell exit) and the following phases (cell entrance). For CCP-ODEs, sensitivity indices become approximately constant after 10h, while CCP-DDEs' sensitivity indices do not follow any particular pattern and CCP-PBMs seem to keep their indices relatively constant and with a lower value. 


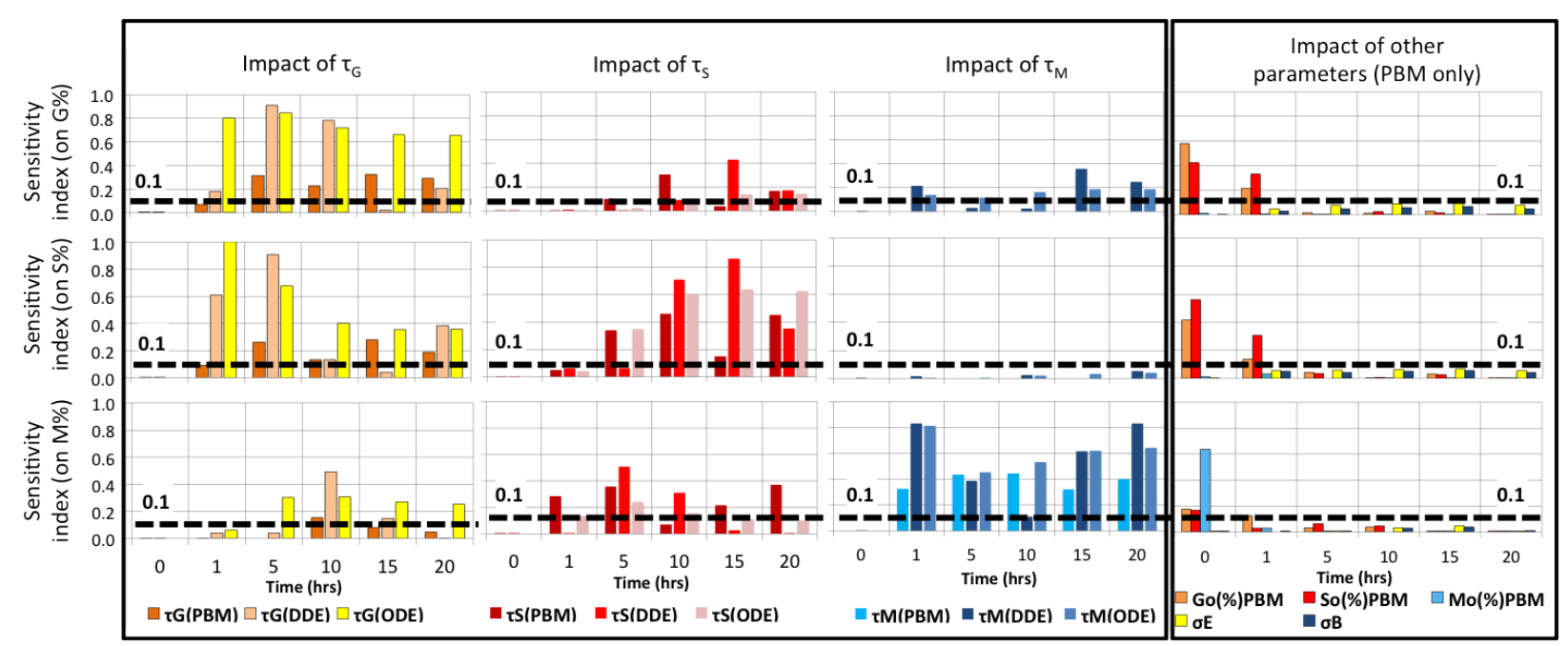

Figure 3: GSA sensitivity indices of phase fractions $\mathrm{G} \%, \mathrm{~S} \%$ and $\mathrm{M} \%$ to phase duration parameters $\tau_{G}, \tau_{S}$ and $\tau_{M}$ in CCP-PBM, CCP-DDEs, CCP-ODEs. The x-axis represents culture time in hours; the y-axis represents the sensitivity indices of each phase fraction: G\% (top), S\% (middle) and M\% (bottom). The indexes are gathered by sensitive phase (rows) and by parameter analyzed $\left(\tau_{G}, \tau_{S}\right.$ and $\tau_{M}$ in each model, initial distribution $(\mathrm{PBM})$ and phase variability (PBM), columns). (MEC-1).

Can the models fit experimental data? To compare the ability of all three models to fit experimental data, we used the synchronous experimental data of Fuentes-Garí et al. ${ }^{23}$ together with the CCP-PBM simulation results reported. Results for all three models can be seen in Figure 4, and parameter estimates (Table 2) for CCP-ODE and CCP-DDE were fitted computationally using the parameter estimation module of gPROMS (Process Systems Enterprise), which utilizes the max log likelihood algorithm (parameters for CCP-PBM were extracted from experiments ${ }^{23}$ ). The CCP-PBM and CCP-DDE presented here can capture synchronous oscillatory behavior while the CCP-ODE fail to account for this intra-phase heterogeneity, resulting in a lack of fit of this particular model, as confirmed by the residual sum of squares (Table 3). Linear stability analysis (LSA) is a model analysis technique that determines systems of equations' dynamic behavior prior to any simulation, by assessing the stability of its steady states. ${ }^{21}$ LSA was performed on CCP-ODE revealing that a decaying amplitude oscillatory behavior is expected for the whole working parameter range (see Part E in the Supporting Information for details). Spatial (such as protein expression in CCP-PBM) 
or temporal (such as the delay history in CCP-DDE) distributions easily facilitate capturing oscillations computationally. The fitted model parameters are always lower for CCP-DDE and higher for CCP-PBM. For CCP-ODE, the fitted parameters do not follow any specific patterns, but in general are closer to the ones of CCP-PBM. Overall difference between values outside the experimental range is $2 \mathrm{~h}$ for CCP-PBM, 10.3h for CCP-DDE and $4.7 \mathrm{~h}$ in CCP-ODE (refer to Table 2). Therefore, the CCP-PBM cell cycle times are consistently the closest to the ones measured experimentally, which together with the model's structure strengthens the CCP-PBM's ability to capture the underlying biology in a more meaningful way.

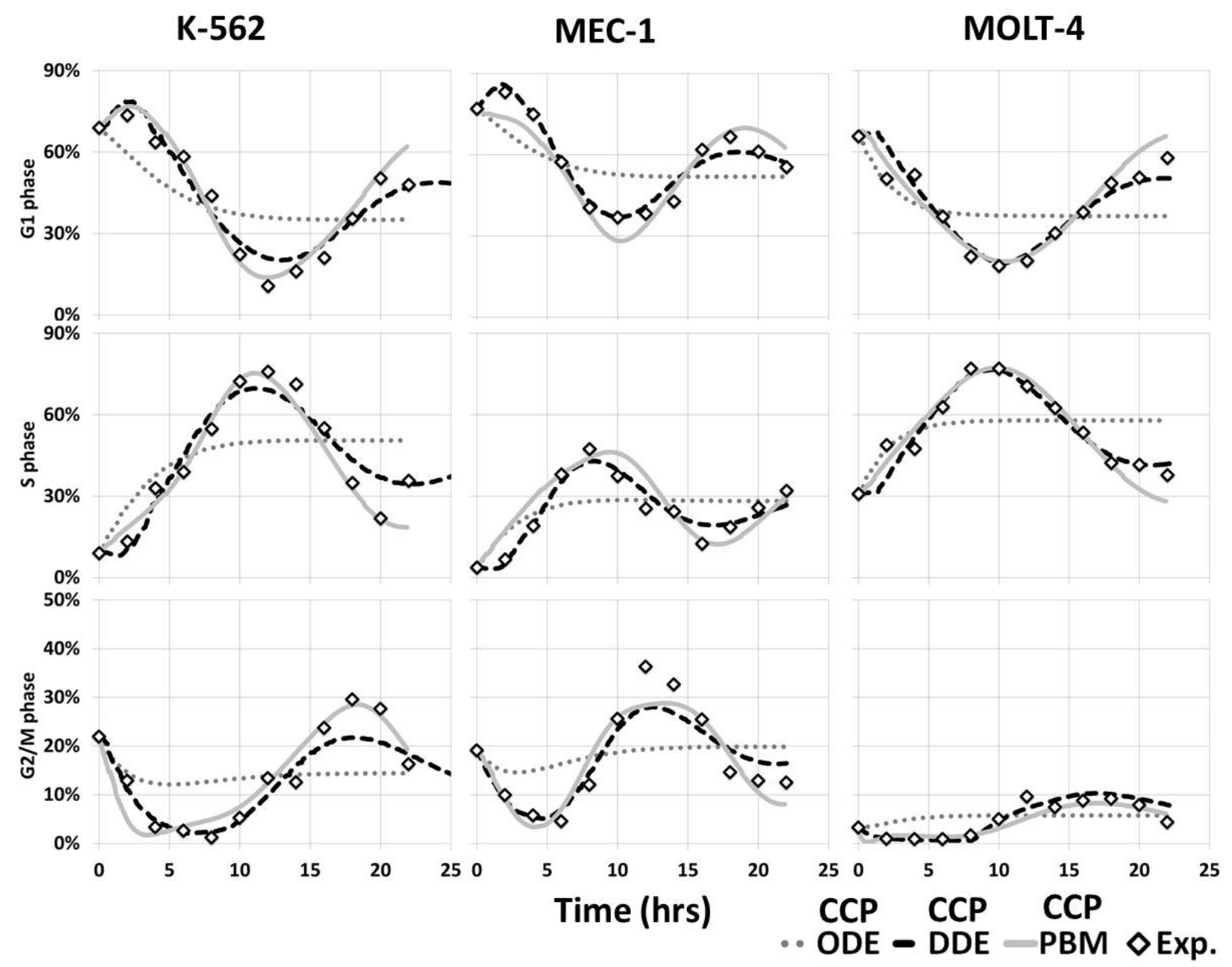

Figure 4: Comparison of experimental phase percents in 3 leukemia cell lines (K-562, MEC-1, MOLT-4) with simulation results for CCP-ODE, CCP-DDE, CCP-PBM. 
Table 2: Phase time parameters estimated in each of the three models as compared to the ones obtained experimentally.

\begin{tabular}{c|c|c|c|c|c} 
Cell line & CC phase time & CCP-PBM & CCP-DDE & CCP-ODE & Exp. measurements \\
\hline \multirow{3}{*}{ K-562 } & $\tau_{G}$ & $9.0 \mathrm{~h}$ & $4.4 \mathrm{~h}$ & $5.8 \mathrm{~h}$ & $6 \pm 2 \mathrm{~h}$ \\
& $\tau_{S}$ & $10.0 \mathrm{~h}$ & $6.6 \mathrm{~h}$ & $12.0 \mathrm{~h}$ & $10 \pm 2 \mathrm{~h}$ \\
& $\tau_{M}$ & $5.0 \mathrm{~h}$ & $2.7 \mathrm{~h}$ & $3.9 \mathrm{~h}$ & $4 \pm 2 \mathrm{~h}$ \\
\hline \multirow{3}{*}{ MEC-1 } & $\tau_{G}$ & $8.5 \mathrm{~h}$ & $4.6 \mathrm{~h}$ & $8.4 \mathrm{~h}$ & $7 \pm 2 \mathrm{~h}$ \\
& $\tau_{S}$ & $7.5 \mathrm{~h}$ & $2.9 \mathrm{~h}$ & $5.6 \mathrm{~h}$ & $8.5 \pm 2 \mathrm{~h}$ \\
& $\tau_{M}$ & $3.0 \mathrm{~h}$ & $2.6 \mathrm{~h}$ & $4.7 \mathrm{~h}$ & $5 \pm 2 \mathrm{~h}$ \\
\hline \multirow{2}{*}{ MOLT-4 } & $\tau_{G}$ & $12.0 \mathrm{~h}$ & $5.0 \mathrm{~h}$ & $4.2 \mathrm{~h}$ & $10 \pm 2 \mathrm{~h}$ \\
& $\tau_{S}$ & $15.0 \mathrm{~h}$ & $8.5 \mathrm{~h}$ & $10.3 \mathrm{~h}$ & $12 \pm 2 \mathrm{~h}$ \\
& $\tau_{M}$ & $2.0 \mathrm{~h}$ & $1.4 \mathrm{~h}$ & $1.1 \mathrm{~h}$ & $2 \pm 2 \mathrm{~h}$
\end{tabular}

In summary, CCP-ODE do not capture the short-term oscillatory behavior required; CCP-DDE capture it well but its parameters do not correlate to the underlying biology; finally, CCP-PBM produce both the oscillations required and parameters in the range of the ones found experimentally.

Table 3: Residual sum of squares (RSS) for the parameter estimation in each model, for each cell line.

\begin{tabular}{c|c|c|c} 
Cell line & CCP-PBM & CCP-DDE & CCP-ODE \\
\hline K-562 & 0.1017 & 0.1038 & 0.6527 \\
MEC-1 & 0.1046 & 0.0461 & 0.3550 \\
MOLT-4 & 0.0488 & 0.0468 & 0.3906
\end{tabular}

\section{Satisfying short-term and long-term kinetics}

The previous section showed that transient kinetics for 1 cell cycle is successfully captured by both CCP-DDE and CCP-PBM, albeit with parameter discrepancies compared to the actual experimental measurements for CCP-DDE. For a short-term scenario (1 cell cycle), cell cycle durations sufficiently simulate phase kinetics; alternatively, steady state parameters may be used to calculate them (see Part D in the Supporting Information). Phase variabilities can be set at their nominal level since they are not significant in the short-term. If protein kinetics is also required, then minima and thresholds for each of them have to be determined for the CCP-PBM. This was further confirmed by the GSA results. ${ }^{23}$ However, transient kinetics 
provides only partial information, as steady state behavior represents a major component of leukemia growth in the resting periods between chemotherapy cycles.

Defining the oscillatory behavior required The oscillatory behavior is characterized by its time to steady state, value at the steady state and oscillation amplitude (all observable experimentally, Figure 5), for each of the phases. It is worth mentioning that previous steady state analyses performed on one compartment models have determined analytically the rate at which oscillations decay over time. The exponential rate at which oscillations decay has not been found in models similar to CCP-PBM with three distributed compartments. ${ }^{32}$ However, numerical simulations can be run for specific cases and relevant parameters such as the amplitude of the oscillations or the time to steady state can be obtained.

The equations reproduced here are for $G$ phase but equations for $S$ and $M$ would be obtained similarly. The time to steady state $\left(T_{S S, G}\right)$ is defined as the time it takes for oscillations to dampen to a level lower than a certain tolerance $\epsilon$, or:

$$
\frac{\left|G_{S S, \%}-G_{\text {peak }, \%}\left(T_{S S, G}\right)\right|}{G_{S S, \%}}<\epsilon
$$

As a standard, $\epsilon=1 \%$ will be used in the simulations subsequently, since the experimental error is always greater than $1 \%$ and does not allow identifying oscillations under that threshold.

The value at steady state is calculated as discussed in the Model Definitions Section, while the amplitude of the oscillations $\left(A_{G}\right)$ represents the deviation from the steady state value at each of the peaks:

$$
A_{G}=\frac{\left|G_{S S, \%}-G_{\text {peak }, \%}(t)\right|}{G_{S S, \%}}
$$

In order to satisfy oscillations at all three levels mathematically, three degrees of freedom per phase are required. 
Can short-term and long-term kinetics be concurrently satisfied? For CCP-DDE and CCP-ODE, all parameters have already been utilized for the initial dynamics; they do not have enough degrees of freedom to validate both the transient state and the steady state and will have a significant limitation tackling long-term dynamic problems. Adjusting oscillatory behavior at all dimensions $\left(T_{S S, G}, T_{S S, S}, T_{S S, M}, G_{S S, \%}, S_{S S, \%}, M_{S S, \%}, A_{G}, A_{S}\right.$ and $\left.A_{M}\right)$ is only possible in CCP-PBM. Up to now, only 2 parameters per phase have been exploited: phase times and initial conditions. However, there is a parameter that did not appear significant in the first few hours in PBM, but has an important influence in those oscillatory properties later on: the standard deviation for the transitions in G and M. In fact, it is the interplay between phase duration and variability in the transition that defines the CCP-PBM's oscillatory characteristics. Different values of $\tau_{G}$ and $\sigma_{E}$ were tested (all else being equal) based on MEC-1 parameters; results are shown in Figure 5. Increasing variability decreases $T_{S S}, G_{S S}$ and $A_{G}$ (after the first oscillation), while resulting in a more pronounced first oscillation. Conversely, increasing $\tau_{G}$ does not have such a significant impact on $T_{S S}$, with increased variation in $G_{S S}$, and a clear difference in $A_{G}$ for the first oscillation (moderately later). Finally, higher deviations from $G_{S S}$ in the initial phase percents result in longer $T_{S S}$ and larger $A_{G}$, but no effect is seen in the actual value $G_{S S}$ (as expected since the initial conditions do not appear in the steady state equations).

What parameters are needed long-term? To accurately define the parameters needed long term, we performed a GSA on the CCP-PBM for $500 \mathrm{~h}$, varying the phase times $\left(\tau_{G}, \tau_{S}\right.$ and $\left.\tau_{M}\right)$ and phase variabilities $\left(\sigma_{E}\right.$ and $\left.\sigma_{B}\right)$. The initial conditions appear to be significant only in the beginning of the transient state; as a result, they will not be included in this analysis which focusses on the steady state. The sensitivity of the phase fraction amplitudes $\left(A_{G}, A_{S}\right.$ and $\left.A_{M}\right)$ was monitored over time, while the sensitivity of the steady state variables $\left(G_{S S, \%}, S_{S S, \%}\right.$ and $\left.\alpha\right)$ and the time to steady state $\left(T_{S S}\right)$ were recorded at the end of the $500 \mathrm{~h}$ period (when all dynamic simulations had reached steady state). 
The GSA results reported in Figure 6 reveal that G2/M phase parameters are not very relevant to the overall oscillatory behavior (it is the shortest phase by hours). Next, we see that $\tau_{S}$ is very significant for $A_{G}$ and $A_{M}$ initially but decreases with time, as $\tau_{G}$ increases mildly. $\tau_{G}$ is equally significant for $A_{S}$ and decreases only slightly with time. The effect of variables from previous phases on the entrance of cells is confirmed long-term (for G0/G1, it is $\tau_{S}$ that becomes relevant since $\mathrm{G} 2 / \mathrm{M}$ is very short). Additionally, $\sigma_{E}$ becomes more significant in the long-term (although its sensitivity index (SI) stays below the one of $\tau_{G}$ ). Therefore, the amplitude of the oscillations depends partly on the variability in $G$ phase, which was also observed in cell automata models ${ }^{33}$ and experimentally. ${ }^{34}$ To summarize, the amplitude of the oscillations is controlled by phase durations at earlier times and by a balance of phase durations and phase variability at later times. For the steady state values, only $\tau_{G}$ and $\tau_{S}$ appear significant (sensitivity index $\geq 0.1$ ). Surprisingly, for $T_{S S}$, only $\tau_{S}$ is important.

\section{Can the necessary parameters be measured?}

The necessary parameters for CCP-DDE and CCP-ODE are the initial conditions and either the steady state distribution + log-growth coefficient ("steady state parameters") or the phase times ("dynamic parameters"). CCP-PBM will require additionally the transition variabilities for G and M (Figure 7).

Obtaining dynamic parameters experimentally requires labelling a cellular subset (S phase cells which incorporate the DNA label during the duplication process) in culture and tracking its phase-to-phase movement over one cell cycle; this is because natural asynchrony makes it impossible to watch cells move in a unified manner between phases. State variable levels (cyclins $\mathrm{E}$ and $\mathrm{B}$, and DNA content) are monitored in parallel in order to seamlessly validate CCP-PBM's phase progression features. Tracking typically occurs over the course of one cycle (20-40hrs for cell lines) and needs a minimum sampling frequency to shape phase oscillations $(2-4 \mathrm{hrs})$. Cell cycle times $\left(\tau_{G}, \tau_{S}\right.$ and $\left.\tau_{M}\right)$ are extracted directly 

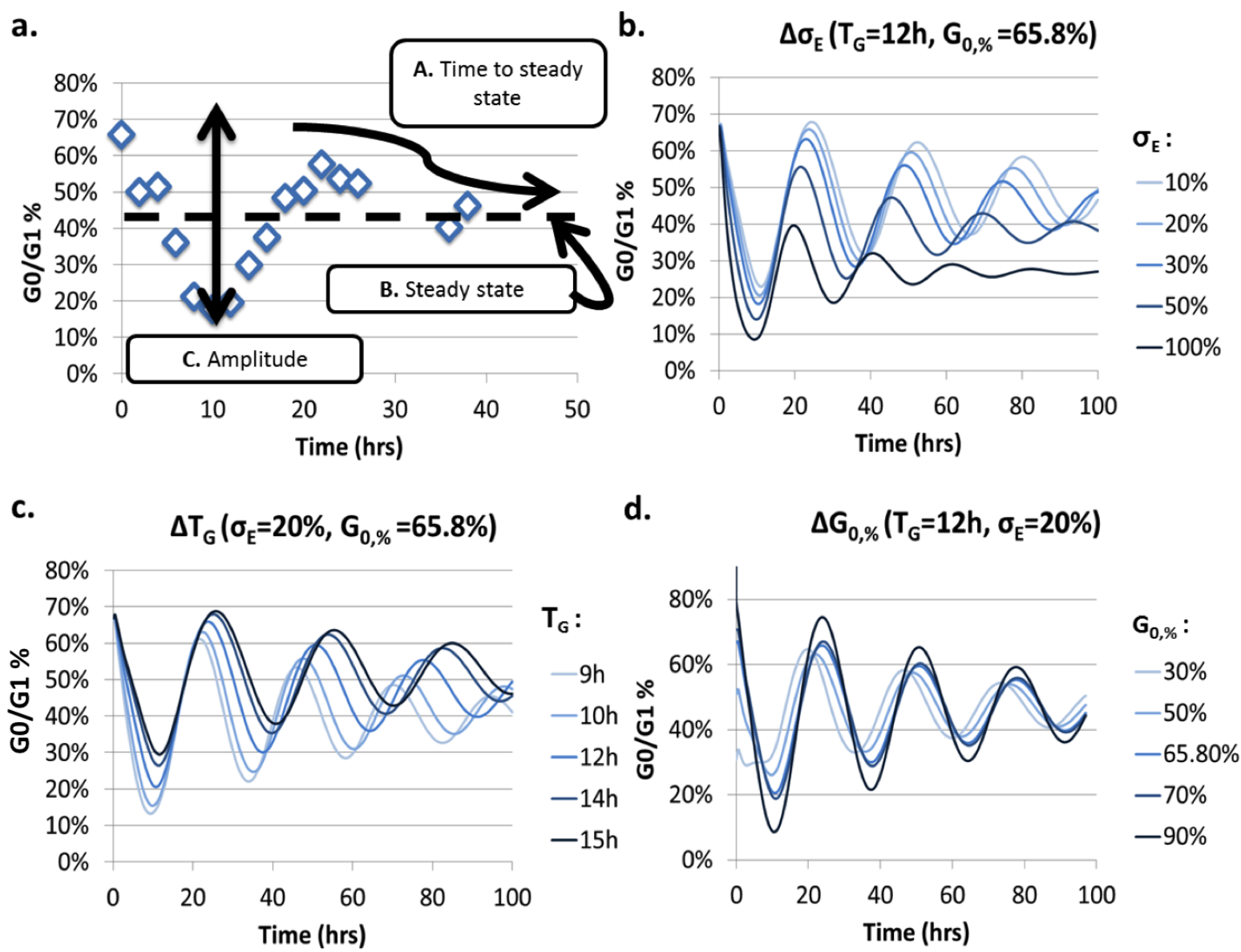

Figure 5: Capturing oscillatory properties: a. experimental evolution of G0/G1 \% (MOLT-4 cell line) over 40h, vs CCP-PBM output under different scenario: b. varying the standard deviation of the transition probability; $\mathbf{c}$. varying the phase time; or $\mathbf{d}$. varying the initial phase fraction (all else being equal). 


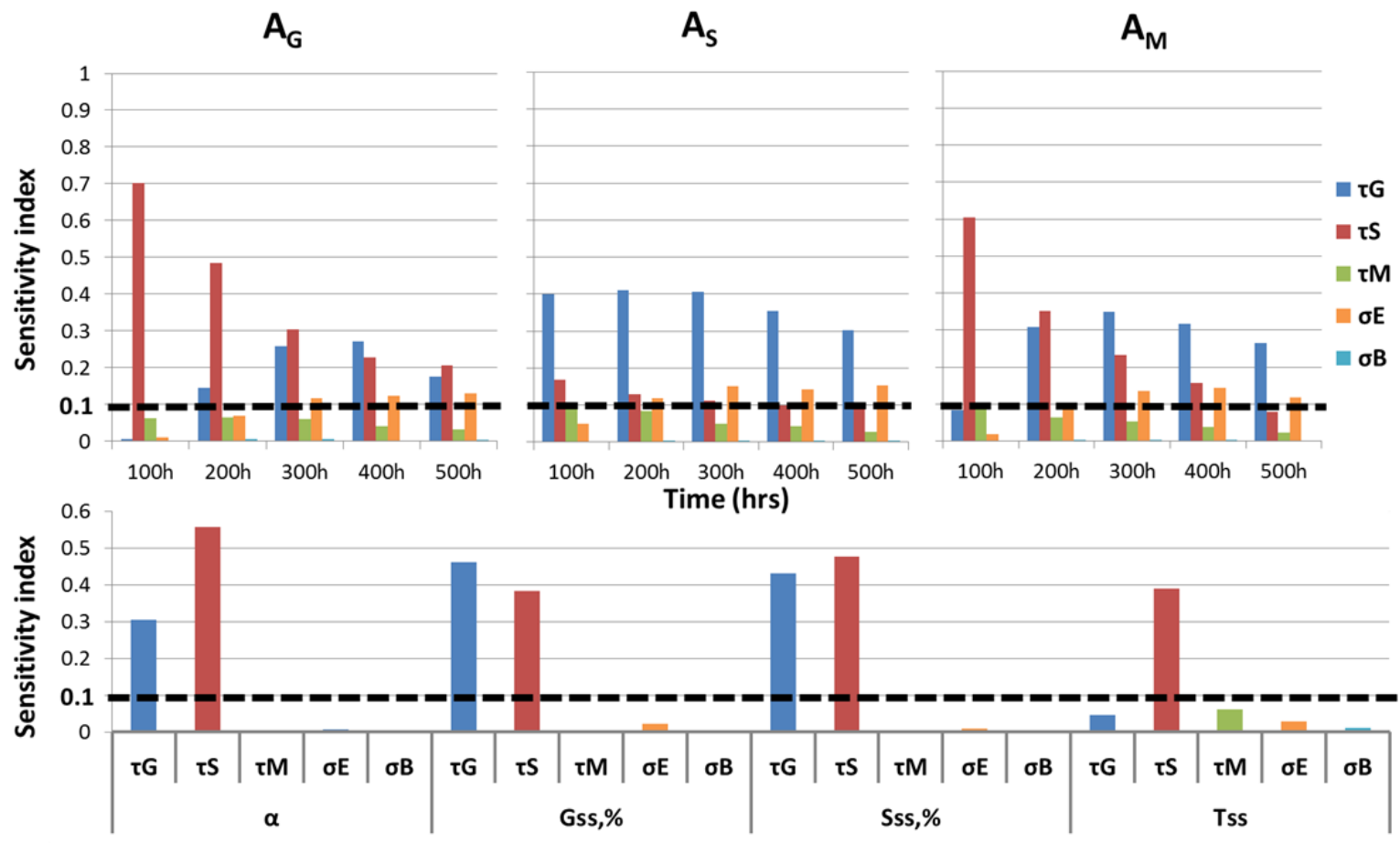

Figure 6: Global sensitivity analysis of CCP-PBM oscillations over 500h (MEC-1), varying phase times and variabilities. Outputs monitored are the amplitude of the oscillations in each phase (top 3 panels) and the steady state variables (at 500h).
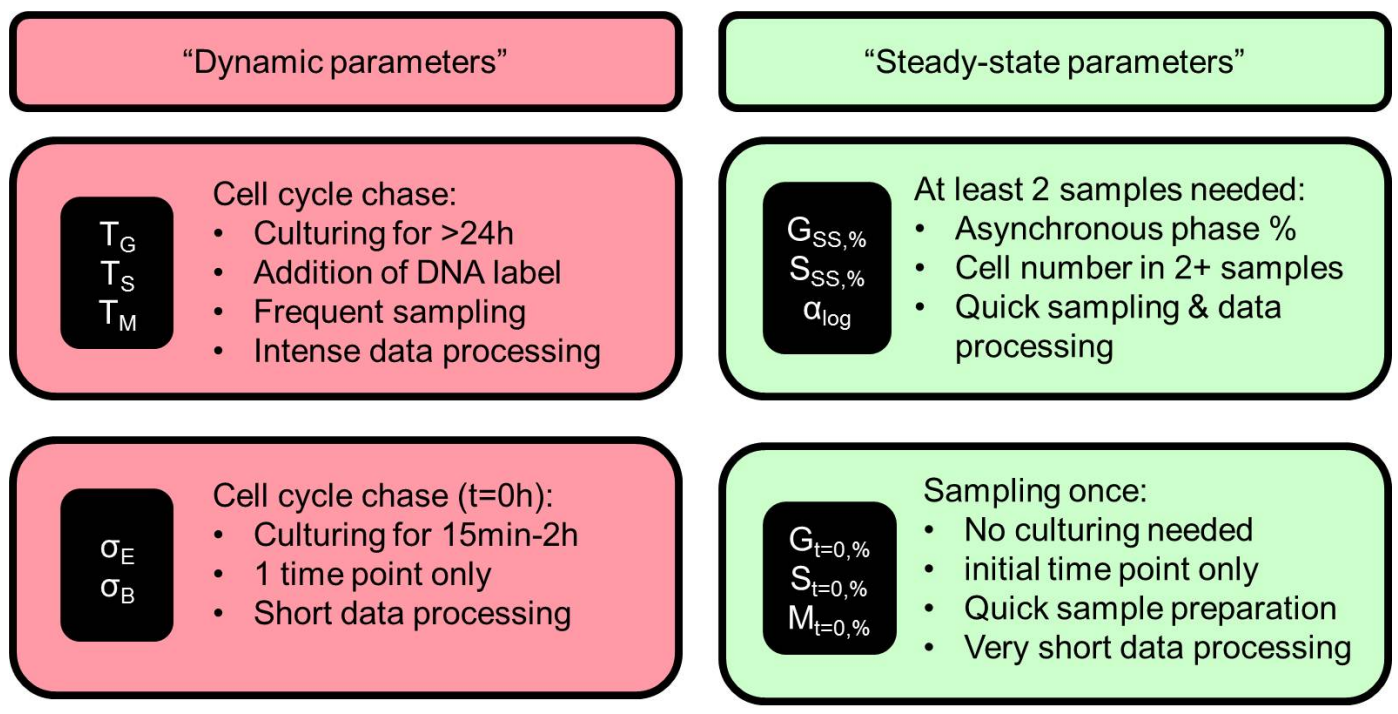

Figure 7: Experimental protocols to carry out in order to obtain each of the parameter sets. "Dynamic parameters" require culturing cells for some period of time, while "steady state parameters" can be obtained directly from any samples. 
from experimental data, and then the model can be validated against the dynamic phase evolution.

In contrast, steady state parameters, as their name suggests, need only selected static data to be determined. For the steady state phase distribution $\left(G_{S S, \%}, S_{S S, \%}, M_{S S, \%}\right)$, a single sample (in exponential growth conditions) is sufficient, as the experimental measurement itself gives directly the phase fractions. For the log-growth parameter $(\alpha, \gamma$ or $\mu$, depending on the model), at least two samples from different times (taken from the same unchanged culture) are needed. However, increased sample numbers give a higher accuracy for both parameter types. Typically, these measurements can be obtained in parallel with others (no need for cell cycle-specific experiments) and during convenient times (not limited to specific times within one cell cycle). As a downside, the data obtained is insufficient to reliably validate the simulations.

In vitro, any of the two parameter sets can be obtained, albeit with significantly different experimental effort. Because both parameter sets are linked through the steady state equations (Part D of the Supporting Information), they can be used interchangeably in silico from a theoretical point of view.

\section{Analysis under chemotherapy treatment}

\section{Setting the grounds for comparison}

We have established that CCP-ODE and CCP-DDE do not have the required degrees of freedom per phase (2) to capture the oscillatory features required (3). In order to continue with the comparison, a choice must be made: either to satisfy the steady-state constraints or the transient behavior. If we choose to satisfy dynamic constraints (lacking degrees of freedom), only short term, transient kinetics will be well captured. On the contrary, if steady state constraints are satisfied, total cell kinetics and cell cycle distribution will be correctly simulated long-term, at the expense of initial dynamics. The decision should be taken based on the application envisaged: since the purpose of cell cycle models is to capture the response 
to chemotherapy (long-term process that starts at steady state, highly dependent on total cell number), ensuring an accurate steady state response is critical. Based on this choice, and for CCP-PBM/CCP-DDE/CCP-ODE comparisons to be fair, the total kinetics (so that the cell number is equal) and the cell cycle distribution (so that the percentage of cells likely to be affected when chemotherapy starts) will have to be equal at steady state.

Equaling $\alpha=\gamma=\mu(\mathrm{Eq}(7)-(9))$ and two of the phase fractions $\left(G_{S S, \%, \mathrm{CCP}-\mathrm{ODE}}=\right.$ $\left.G_{S S, \%, \mathrm{CCP}-\mathrm{DDE}}=G_{S S, \%, \mathrm{CCP}-\mathrm{PBM}} ; S_{S S, \%, \mathrm{CCP}-\mathrm{ODE}}=S_{S S, \%, \mathrm{CCP}-\mathrm{DDE}}=S_{S S, \%, \mathrm{CCP}-\mathrm{PBM}}\right)$, cell cycle times can be converted from one system (CCP-ODE, CCP-DDE, CCP-PBM) to another. Given the cell cycle times in the CCP-DDE system (which is the most complex to solve practically), all other cell cycle times can be obtained through this process of equaling the total growth rate and the phase distributions at steady state (see sample parameter conversion in Table 4).

Table 4: Cell cycle times converted from CCP-DDE to CCP-PBM and CCP-ODE for the steady state conditions chosen $\left(G_{S S, \%}=79.3 \%, S_{S S \%}=16.0 \%, M_{S S \%}=4.8 \%, \alpha=\mu=\right.$ $\left.\gamma=0.0157 h^{-1}\right)$

\begin{tabular}{c|c|c|c} 
& CCP-PBM & CCP-DDEs & CCP-ODEs \\
\hline$\tau_{G}$ & $36.8 \mathrm{~h}$ & $16.6 \mathrm{~h}$ & $41 \mathrm{~h}$ \\
$\tau_{S}$ & $8.9 \mathrm{~h}$ & $4.7 \mathrm{~h}$ & $10 \mathrm{~h}$ \\
$\tau_{M}$ & $3.4 \mathrm{~h}$ & $1.5 \mathrm{~h}$ & $3 \mathrm{~h}$
\end{tabular}

\section{How sensitive is the model to chemotherapy effects?}

We have seen that both CCP-PBM and CCP-DDE are capable of fitting synchronous experimental data, a fact that is critical in modeling chemotherapy due to its phase-specific action (G1 and S phase) and subsequent aggregation of cells into synchronized populations. ${ }^{8}$ In this section, each model will be embedded into a PK/PD model of chemotherapy delivery in leukemia ${ }^{20}$ and tested for its sensitivity to chemotherapy (as captured by the chemotherapy drug effect parameters $k_{D, S}$ and $k_{D, G}$ for $\mathrm{S}$ and G1 phases respectively in CCP-ODE-PD, CCP-DDE-PD and CCP-PBM-PD). 
An example male patient that is $167.5 \mathrm{~cm}$ tall and $79.3 \mathrm{~kg}$ in weight, 80 years old, receiving a chemotherapy treatment of $100 \mathrm{mg} / \mathrm{m}^{2}$ Ara-C (12h infusion twice a day for 10 days) and $50 \mathrm{mg} / \mathrm{m}^{2}$ DNR (pulse dose on days 1, 3 and 5) is selected for a PK/PD comparison. The latter are the input parameters for the PK model.

Comparing total cell evolution over the treatment period in the three models (Figure 8), we observe that the CCP-ODE-PD sensitivity to chemotherapy is effectively lower due to its inherent tendency to allow the passage of cells from phase to phase (Figure 9). Its absence of intra-phase distribution results in a fraction of the cells in $\mathrm{S}$ always eligible to move to the next phase. Conversely, CCP-DDE-PD and CCP-PBM-PD allow for cells to be distributed
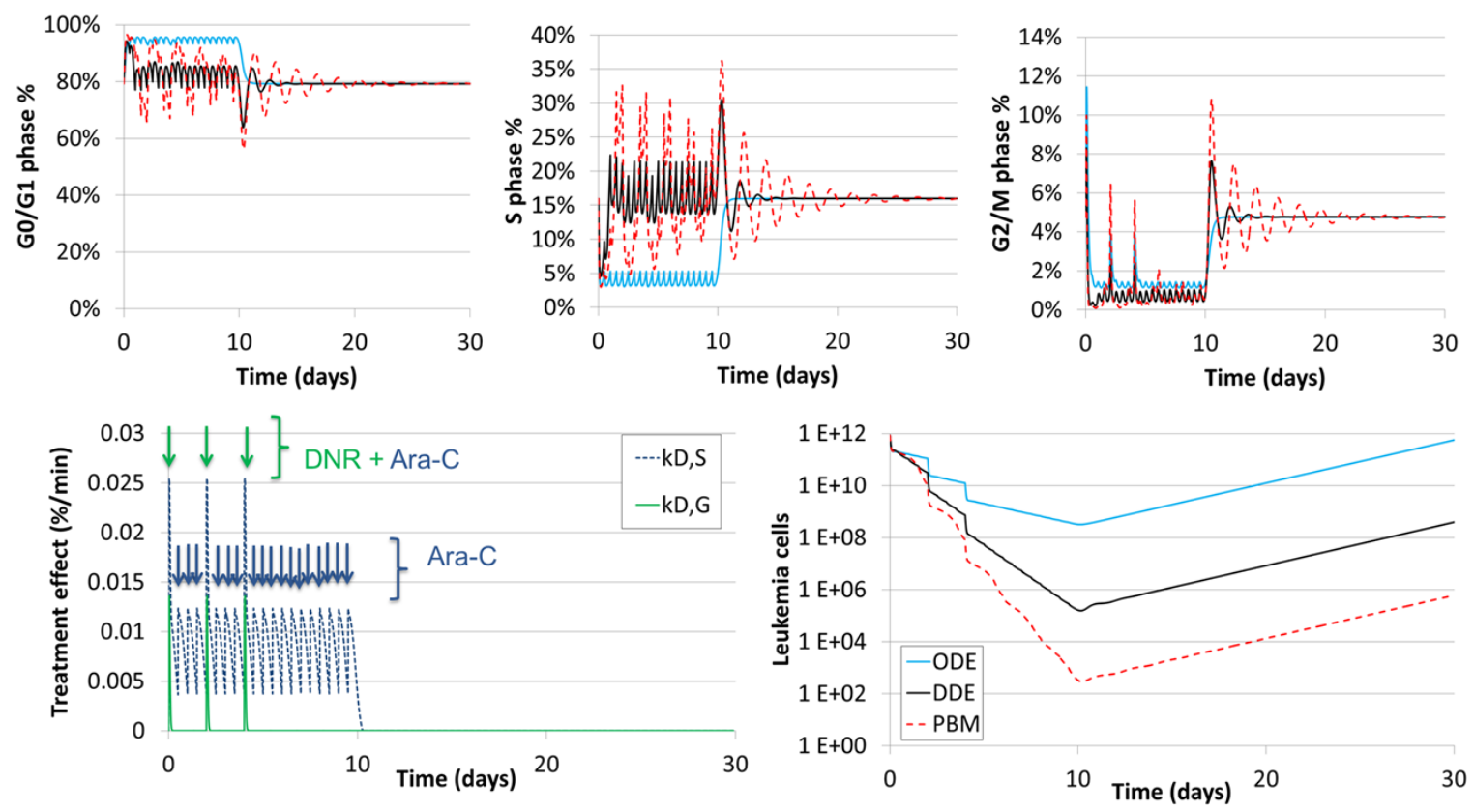

Figure 8: Comparison of cell cycle distribution (top 3 panels) and total cell number(bottom right) under chemotherapy treatment (bottom left panel) in: CCP-ODE-PD, CCP-DDE-PD, CCP-PBM-PD under equivalent growth kinetics (converted from CCP-DDE-PD to equivalent parameters in CCP-ODE-PD and CCP-PBM-PD - see equal cell cycle distribution and total growth kinetics after 10 days of treatment)

in each phase (temporally and spatially, respectively), creating a downward trend in the percentage of cells in the distribution. This is in accordance with the intuitive fact that subjecting cells to drug effects throughout a phase will make it less likely to find cells at the end of the phase. As a result, fewer cells are found in CCP-DDE-PD and CCP-PBM-PD 
in G2/M (Figure 8). CCP-ODE-PD reach higher G2/M phase percents, representing the higher fraction of $\mathrm{S}$ phase cells coming in. The percentage of G2/M cells becomes critical due to its impact on total cell duplication (if cells do not reach mitosis, duplication cannot occur).

Drug sensitivity is reflected in the slope (logarithmic) of the decrease in the total leukemia cells: moderate for CCP-ODE-PD, intermediate for CCP-DDE-PD and steep for CCPPBM. CCP-DDE-PD response seems to be halfway between CCP-PBM-PD and CCP-ODEPD, in that cell totals are 2-3 orders of magnitude above CCP-PBM-PD and below CCPODE-PD, while the cell cycle distribution oscillatory behavior can be broadly said to be a combination of CCP-ODE-PD (peak timings) and CCP-PBM-PD (central value). Note that CCP-PBM-PD and CCP-DDE-PD fluctuate approximately around the steady state value for $\mathrm{S}$ and G0/G1 fractions during treatment, while CCP-ODE-PD remain at a high for $G_{\%}$, compensated by a low $S_{\%}$. Oscillations post-treatment are the most sustained for CCP-PBM-PD, while CCP-DDE-PD take 1-2 cell cycles to stabilize and CCP-ODEPD immediately reach steady state. Further, when changing the $\sigma / \tau$ balance in G0/G1 and G2/M for the CCP-PBM-PD (always maintaining the same steady state), oscillations decrease in amplitude when increasing $\sigma$ (Figure 10) and may converge to a shape similar to the CCP-DDE-PD oscillations (see $\sigma=50 \%$ ). Similarly, totals become lower at the end of treatment with decreasing $\sigma$.

Cojoracu and Agur ${ }^{35}$ obtained a similar result related to increased resistance of higher standard deviations of phase durations. Even with the highest variability tested $(\sigma=50 \%)$, the total number at the end of treatment (day 10) is lower than the one for CCP-DDEPD (despite the cycle fractions being very similar). The simple fact that the CCP-PBMPD's oscillatory behavior can be adjusted allows for the possibility of taking into account these discrepancies in treatment response. We admit CCP-DDE-PD could benefit from adding phase duration variability (or decoupling the transition parameters from the delays), requiring an extra parameter per phase and thus allowing increased flexibility in fitting 


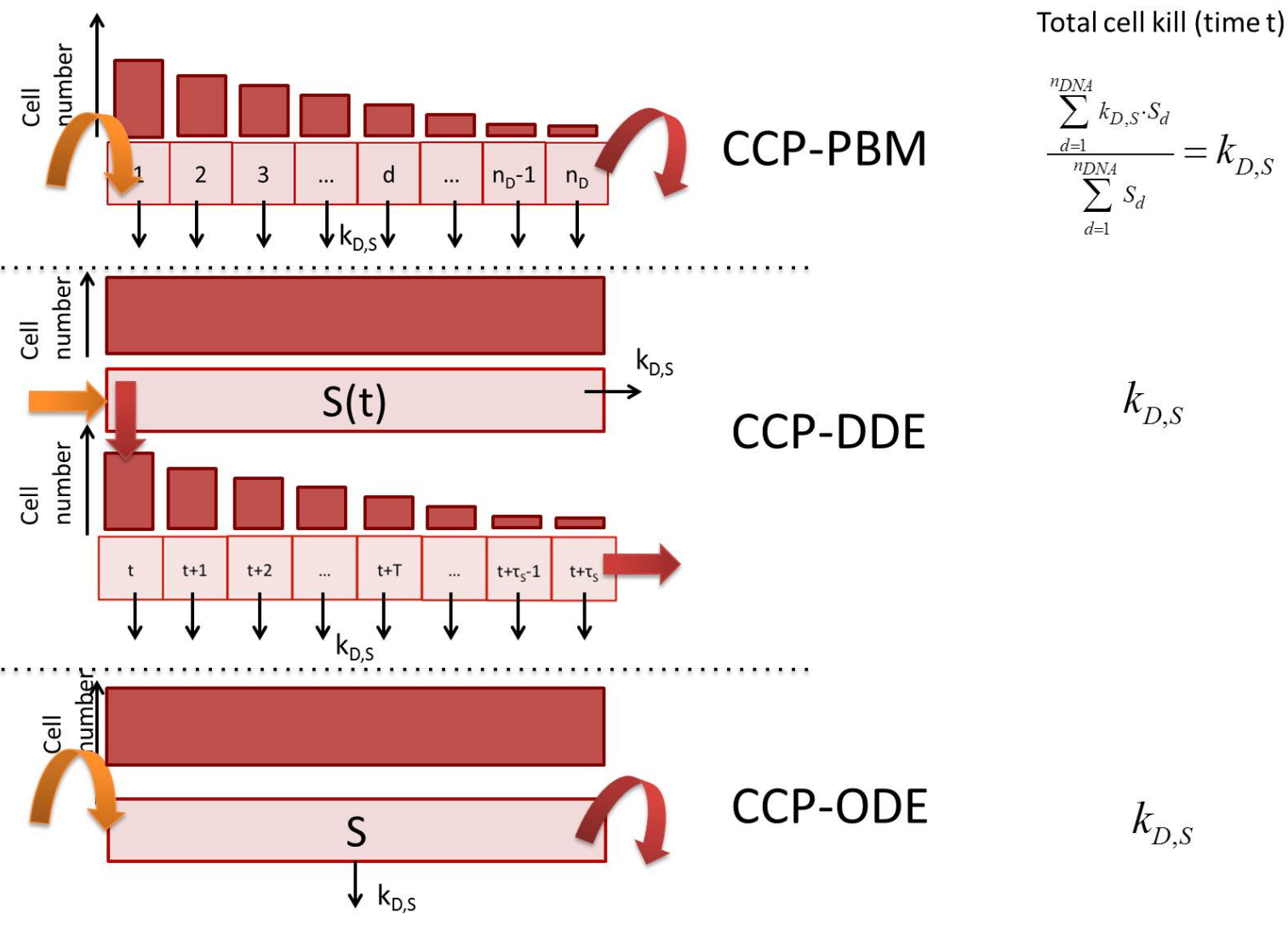

Figure 9: Model mechanics under chemotherapy effects. Observe that total cell kill at time $\mathrm{t}$ is the same, however the drug effect bin after bin (for CCP-PBM-PD, or time after time, for CCP-DDE-PD) results in decreasing percentages of the population that initially entered the phase reaching the final bins (for CCP-PBM-PD, or times for CCP-DDE-PD). 
oscillatory patterns. Nevertheless, the superiority of CCP-PBM-PD lies in its biological relevance, which allows direct parameter measurement and validation. Significant differences have been observed in model behavior especially when all efforts have been made to make model conditions as close as possible.
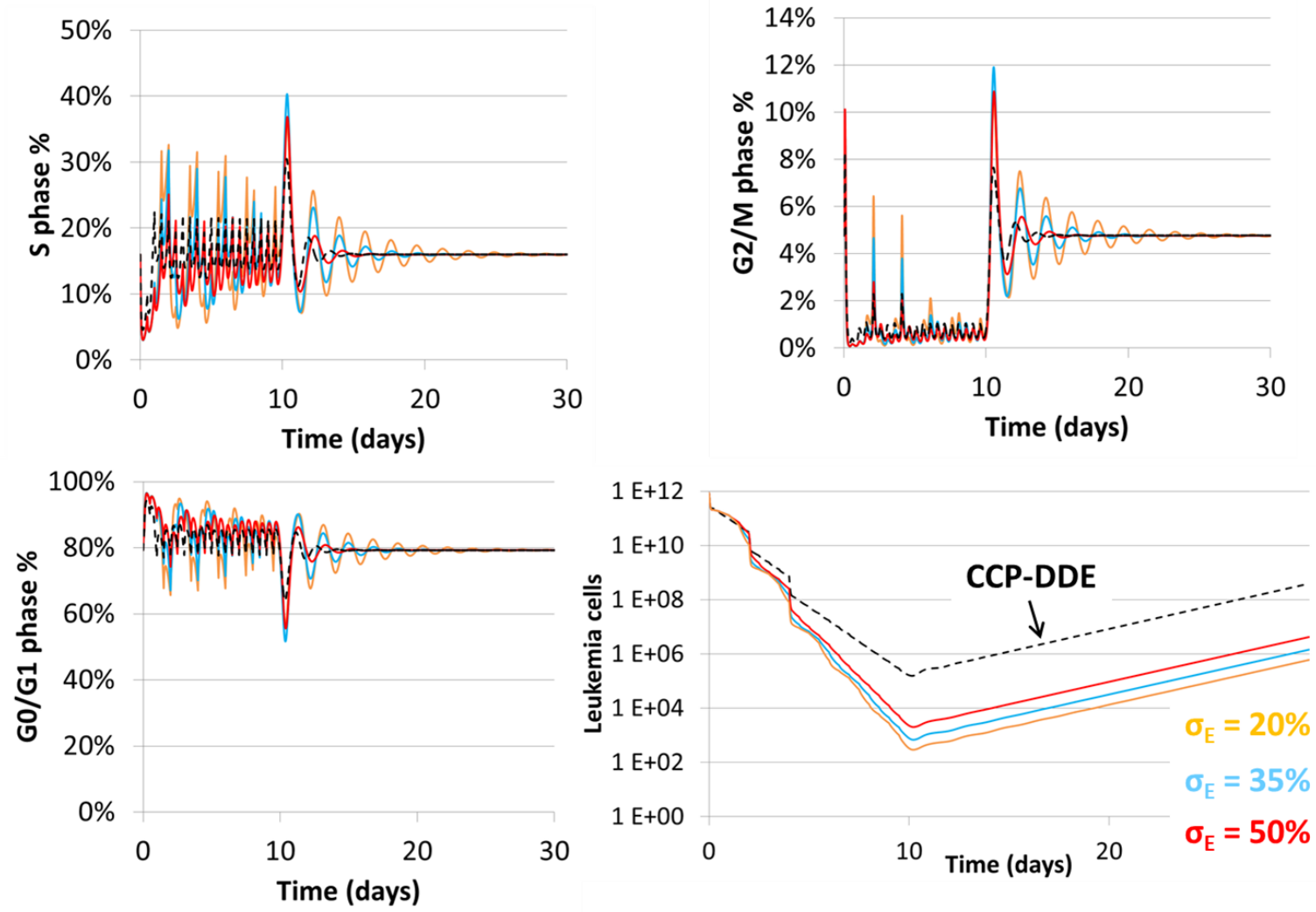

Figure 10: Comparison of cell cycle distribution (top, bottom left) and total cell number (bottom right) under same conditions as Figure 8 in CCP-PBM-PD (change in $\sigma \%$ and $\tau$ according to same steady state) and CCP-DDE-PD (black dashed line) under equivalent growth kinetics

\section{Discussion}

Three different models representing cell cycle kinetics were presented here and analyzed for their ability to capture oscillations and their response to chemotherapy under the same steady state behavior. When fitted to experimental data, only CCP-DDE and CCP-PBM performed well, with CCP-PBM having the most biologically relevant parameters (additionally to ac- 
curately predicting intracellular protein expression as reported in Fuentes-Garí et al. ${ }^{23}$ ). A procedure for converting a set of in vitro measured model parameters to a different set of model parameters that define model dynamics equally well is then presented. An important advantage of this is potentially more efficient experimentation. A second advantage of interconvertibility in parameter sets was homogeneity and univocal definition with respect to expected behavior under PK/PD conditions. Each of the models was then embedded in a PK/PD model of chemotherapy for AML and a patient case was run. Throughout the treatment, CCP-ODE had the highest percent of cells in the G2/M phase and the lowest amount of cell kill, which was explained by their inability to capture intra-phase distributions, letting cells transition to the next phase as soon as they have entered (thus escaping continuous phase exposure to the chemotherapy effect). Conversely, CCP-PBM and CCP-DDE set a spatial or temporal restriction to the eligibility of cells to transition to the next phase, and subject those cells for a minimum phase time to the effect of chemotherapy (leading to a lower percent of G2/M cells and a higher cell kill at the end of the treatment).

Cell cycle model selection has a critical impact on predicted chemotherapy response. Current standards in PK/PD rely on cell cycle models that are not always accurate enough and that have not necessarily been validated. For more advanced use of these models, such as for optimal control of chemotherapy delivery ${ }^{36-38}$ and drug scheduling, ${ }^{10}$ a precise representation of cell cycle processes becomes critical (deconvoluting total growth kinetics into cell cycle phases, capturing intra-phase progression). Cell cycle models for chemotherapy optimisation should be carefully selected and suited to their application. A first and critical consideration is whether the model parameters are relevant in vitro/in vivo and are measurable $^{39}$ (or can be converted into a new set of parameters that are). A second consideration is whether both the dynamic (transient) and steady state behaviors obtained are accurate in representing the underlying biology and clinical response (sensitivity to drugs). Finally, the trade-off between model complexity and matching the required degrees of freedom exhibited by the system needs to be taken into account. 
The CCP-PBM model presented here provides a good trade-off between capturing more complex pathway and protein kinetics, which are normally reported in mechanistic models only, and maintaining a computationally efficient solution representing global cell kinetics, of which the CCP-ODE discussed is a clear example. Importantly, treatment personalization requires defining measurable parameters representing patient heterogeneity. ${ }^{40}$ Our CCPPBM is seamlessly linked to experimentally measurable parameters that have been reported to be highly heterogeneous among AML patients. ${ }^{41,42}$ Due to its distributed structure, our CCP-PBM captures cell cycle oscillatory complexity to a great extent, despite being an aggregate model. Moving to more detailed, experimentally validated models is clearly the way forward.

\section{Acknowledgement}

This work is supported by ERC-BioBlood (\#340719), ERC-Mobile Project (\#226462), by the EU 7th Framework Programme [MULTIMOD Project FP7/2007-2013, \#238013], by the National Institute of Health Research via Northwick Park Hospital R\&D and by the Richard Thomas Leukaemia Research Fund. RM thanks the Royal Academy of Engineering for a Research Fellowship. The authors thank N. Diangelakis and M. Papathanasiou for their assistance in global sensitivity analysis procedures and Dr. C. Colijn for insightful discussions on the construction of the CCP-DDE model.

\section{Supporting Information Available}

Supporting Information Available: (A) DDE handling by a dynamic solver and initialization; (B) CCP-PBM discretization features; (C) Steady state analysis; (D) Linear stability analysis of CCP-DDE; (E) GSA: Sobol's method; (F) DDE construction and conservation aspects. This material is available free of charge via the Internet at http://pubs.acs.org/. 


\section{References}

(1) Lane, S. W.; Scadden, D. T.; Gilliland, D. G. The Leukemic Stem Cell Niche: Current Concepts and Therapeutic Opportunities. Blood 2009, 114, 1150.

(2) Morgan, G. Chemotherapy and the Cell Cycle. Cancer Nurs. Pract. 2003, 2, 27.

(3) Kastan, M. B.; Bartek, J. Cell-Cycle Checkpoints and Cancer. Nature 2004, 432, 316.

(4) Sherr, C. J. Cancer Cell Cycles. Science 1996, 274, 1672.

(5) Anderson, A. R. A.; Quaranta, V. Integrative Mathematical Oncology. Nat. Rev. Cancer 2008, 8, 227.

(6) Weis, M. C.; Avva, J.; Jacobberger, J. W.; Sreenath, S. N. A Data-Driven, Mathematical Model of Mammalian Cell Cycle Regulation. PLoS ONE 2014, 9, e97130.

(7) Singhania, R.; Sramkoski, R. M.; Jacobberger, J. W.; Tyson, J. J. A Hybrid Model of Mammalian Cell Cycle Regulation. Plos Comput. Biol. 2011, 7, e1001077.

(8) Sherer, E.; Hannemann, R. E.; Rundell, A.; Ramkrishna, D. Analysis of Resonance Chemotherapy in Leukemia Treatment via Multi-Staged Population Balance Models. J. Theor. Biol. 2006, 240, 648.

(9) Daukste, L.; Basse, B.; Baguley, B. C.; Wall, D. J. N. Mathematical Determination of Cell Population Doubling Times for Multiple Cell Lines. Bull. Math. Biol. 2012, 74, 2510.

(10) Florian Jr., J. A.; Eiseman, J. L.; Parker, R. S. Accounting for Quiescent Cells in Tumour Growth and Cancer Treatment. Syst. Biol. 2005, 152, 185.

(11) García Münzer, D. G.; Kostoglou, M.; Georgiadis, M. C.; Pistikopoulos, E. N.; Mantalaris, A. Cyclin and DNA Distributed Cell Cycle Model for GS-NSO Cells. PLoS Comput. Biol. 2015, 11, e1004062. 
(12) Gardner, S. N. Cell Cycle Phase-Specific Chemotherapy: Computation Methods for Guiding Treatment. Cell Cycle 2002, 1, 369.

(13) Bogle, I. D. L.; Allen, R.; Sumner, T. The Role of Computer Aided Process Engineering in Physiology and Clinical Medicine. Cout. Chem. Eng. 2010, 34, 763.

(14) Swierniak, A.; Kimmel, M.; Smieja, J. Mathematical Modeling as a Tool for Planning Anticancer Therapy. Eur. J. Pharmacol. 2009, 625, 108.

(15) Di Ventura, B.; Lemerle, C.; Michalodimitrakis, K.; Serrano, L. From In Vivo to In Silico Biology and Back. Nature 2006, 443, 527.

(16) Csete, M. E.; Doyle, J. C. Reverse Engineering of Biological Complexity. Science 2002, 295, 1664.

(17) Velliou, E.; Fuentes-Garí, M.; Misener, R.; Pefani, E.; Rende, M.; Panoskaltsis, N.; Mantalaris, A.; Pistikopoulos, E. N. A Framework for the Design, Modeling and Optimization of Biomedical Systems. Proceedings of the 8th International Conference on Foundations of Computer-Aided Process Design 2014, 34, 225.

(18) Fuentes-Garí, M.; Velliou, E.; Misener, R.; Pefani, E.; Rende, M.; Panoskaltsis, N.; Mantalaris, A.; Pistikopoulos, E. N. A Systematic Framework for the Design, Modeling and Optimization of Personalized Healthcare: Making and Healing Blood. Comput. Chem. Eng. 2015, in press.

(19) Pefani, E.; Panoskaltsis, N.; Mantalaris, A.; Georgiadis, M. C.; Pistikopoulos, E. N. Design of Optimal Patient-Specific Chemotherapy Protocols for the Treatment of Acute Myeloid Leukemia (AML). Comput. Chem. Eng. 2013, 57, 187.

(20) Pefani, E.; Panoskaltsis, N.; Mantalaris, A.; Georgiadis, M.; Pistikopoulos, E. N. Chemotherapy Drug Scheduling for the Induction Treatment of Patients with Acute Myeloid Leukemia. IEEE Trans. Biomed. Eng. 2014, 24, 24. 
(21) Ferrell, J. E.; Tsai, T. Y. C.; Yang, Q. O. Modeling the Cell Cycle: Why Do Certain Circuits Oscillate? Cell 2011, $144,874$.

(22) Fuentes-Garí, M.; Misener, R.; Pefani, E.; García Münzer, D. G.; Kostoglou, M.; Georgiadis, M. C.; Panoskaltsis, N.; Pistikopoulos, E. N.; Mantalaris, A. In 12th International Symposium on Process Systems Engineering and 25th European Symposium on Computer Aided Process Engineering; Krist V. Gernaey, J. K. H., Gani, R., Eds.; Comput. Aided Chem. Eng.; Elsevier, 2015; Vol. 37; p 2159.

(23) Fuentes-Garí, M.; Misener, R.; García-Munzer, D.; Velliou, E.; Georgiadis, M. C.; Kostoglou, M.; Pistikopoulos, E. N.; Panoskaltsis, N.; Mantalaris, A. A Mathematical Model of Subpopulation Kinetics for the Deconvolution of Leukaemia Heterogeneity. J. R. Soc. Interface 2015, 12, 20150276.

(24) García Münzer, D. G.; Kostoglou, M.; Georgiadis, M. C.; Pistikopoulos, E. N.; Mantalaris, A. In 24th European Symposium on Computer Aided Process Engineering; Jiří Jaromír Klemeš, P. S. V., Liew, P. Y., Eds.; Comput. Aided Chem. Eng.; Elsevier, 2014; Vol. 33; p 19.

(25) García Münzer, D. G.; Kostoglou, M.; Georgiadis, M. C.; Pistikopoulos, E. N.; Mantalaris, A. Developing a Cyclin Blueprint as a Tool for Mapping the Cell Cycle in GS-NSO. Biochem. Eng. J. 2013, 81, 97.

(26) Colijn, C.; Mackey, M. C. A Mathematical Model of Hematopoiesis I. Periodic Chronic Myelogenous Leukemia. J. Theor. Biol. 2005, 237, 117.

(27) Colijn, C.; Mackey, M. C. Bifurcation and Bistability in a Model of Hematopoietic Regulation. SIAM J. Appl. Dyn. Syst. 2007, 6, 378.

(28) Ward, J. D.; Yu, C.-C.; Doherty, M. F. Plantwide Operation of Processes with Crystallization. AIChE J. 2007, 53, 2885. 
(29) Hiddemann, W. Cytosine-Arabinoside in the Treatment of Acute Myeloid Leukemia the Role and Place of High-Dose Regimens. Ann. Hematol. 1991, 62, 119.

(30) Gewirtz, D. A. A Critical Evaluation of the Mechanisms of Action Proposed for the Antitumor Effects of the Anthracycline Antibiotics Adriamycin and Daunorubicin. Biochem. Pharmacol. 1999, 57, 727.

(31) Hall, C.; Lueshen, E.; Mosat, A.; Linninger, A. A. Interspecies Scaling in Pharmacokinetics: a Novel Whole-Body Physiologically Based Modeling Framework to Discover Drug Biodistribution Mechanisms In Vivo. J. Pharm. Sci. 2012, 101, 1221.

(32) Begg, R.; Wall, D. J. N.; Wake, G. C. On a Multicompartment Age-Distribution Model of Cell Growth. IMA J. Appl. Math. 2010, 75, 905.

(33) Altinok, A.; Gonze, D.; Levi, F.; Goldbeter, A. An Automaton Model for the Cell Cycle. Interface Focus 2011, 1, 36.

(34) John, P. C. L. The Cell Cycle; Seminar series (Society for Experimental Biology (Great Britain)) ; 10.; Cambridge University Press: Cambridge [Cambridgeshire] ; New York, 1981.

(35) Cojoracu, L.; Agur, Z. A Theoretical-Analysis of Interval Drug Dosing for Cell-CyclePhase-Specific Drugs. Math. Biosci. 1992, 109, 85.

(36) Moradi, H.; Vossoughi, G.; Salarieh, H. Optimal Robust Control of Drug Delivery in Cancer Chemotherapy: A Comparison Between three Control Approaches. Computer Meth. Programs Biomed. 2013, 112, 69.

(37) Chen, T.; Kirkby, N. F.; Jena, R. Optimal Dosing of Cancer Chemotherapy using Model Predictive Control and Moving Horizon State/Parameter Estimation. Comput. Methods Programs Biomed. 2012, 108, 973. 
(38) Dua, P.; Dua, V.; Pistikopoulos, E. N. Optimal Delivery of Chemotherapeutic Agents in Cancer. Comput. Chem. Eng. 2008, 32, 99.

(39) Androulakis, I. P.; Kamisoglu, K.; Mattick, J. S. Topology and Dynamics of Signaling Networks: in Search of Transcriptional Control of the Inflammatory Response. Annu. Rev. Biomed. Eng. 2013, 15, 1.

(40) Li, B.; You, L. Predictive Power of Cell-to-Cell Variability. Quant. Biol. 2013, 1, 131.

(41) Raza, A.; Preisler, H. D.; Day, R.; Yasin, Z.; White, M.; Lykins, J.; Kukla, C.; Barcos, M.; Bennett, J.; Browman, G.; Goldberg, J.; Grunwald, H.; Larson, R.; Vardiman, J.; Vogler, R. Direct Relationship between Remission Duration in Acute MyeloidLeukemia and Cell-Cycle Kinetics - a Leukemia Intergroup Study. Blood 1990, 76, 2191.

(42) Gong, J. P.; Ardelt, B.; Traganos, F.; Darzynkiewicz, Z. Unscheduled Expression of Cyclin-B1 and Cyclin-E in Several Leukemic and Solid Tumor-Cell Lines. Cancer Res. $1994,54,4285$.

\section{Figure captions}

Figure 1: Model structure in CCP-PBM, CCP-DDE, CCP-ODE with discretization intervals for CCP-PBM, history vectors and lumped phase compartments for CCP-DDE and lumped phase compartments only for CCP-ODE.

Figure 2: Schematic of the interactions between PK, PD and cell cycle components of the Pefani et al. model, together with input of patient data.

Figure 3: GSA sensitivity indices of phase fractions G\%, S\% and M\% to phase duration parameters $\tau_{G}, \tau_{S}$ and $\tau_{M}$ in CCP-PBM, CCP-DDEs, CCP-ODEs. The x-axis represents 
culture time in hours; the y-axis represents the sensitivity indices of each phase fraction: G\% (top), S\% (middle) and M\% (bottom). The indexes are gathered by sensitive phase (rows) and by parameter analyzed $\left(\tau_{G}, \tau_{S}\right.$ and $\tau_{M}$ in each model, columns). (MEC-1).

Figure 4: Comparison of experimental phase percents in 3 leukemia cell lines (K-562, MEC-1, MOLT-4) with simulation results for CCP-ODE, CCP-DDE, CCP-PBM.

Figure 5: Capturing oscillatory properties: a. experimental evolution of G0/G1 \% (MOLT-4 cell line) over 40h, vs CCP-PBM output under different scenario: b. varying the standard deviation of the transition probability; c. varying the phase time; or $\mathbf{d}$. varying the initial phase fraction (all else being equal).

Figure 6: Global sensitivity analysis of CCP-PBM oscillations over 500h (MEC-1), varying phase times and variabilities. Outputs monitored are the amplitude of the oscillations in each phase (top 3 panels) and the steady state variables (at 500h).

Figure 7: Experimental protocols to carry out in order to obtain each of the parameter sets. "Dynamic parameters" require culturing cells for some period of time, while "steady state parameters" can be obtained directly from any samples.

Figure 8: Comparison of cell cycle distribution (top 3 panels) and total cell number(bottom right) under chemotherapy treatment (bottom left panel) in: CCP-ODE-PD, CCP-DDE-PD, CCP-PBM-PD under equivalent growth kinetics (converted from CCP-DDE$\mathrm{PD}$ to equivalent parameters in CCP-ODE-PD and CCP-PBM-PD - see equal cell cycle distribution and total growth kinetics after 10 days of treatment).

Figure 9: Model mechanics under chemotherapy effects. Observe that total cell kill at 
time $t$ is the same, however the drug effect bin after bin (for CCP-PBM-PD, or time after time, for CCP-DDE-PD) results in decreasing percentages of the population that initially entered the phase reaching the final bins (for CCP-PBM-PD, or times for CCP-DDE-PD).

Figure 10: Comparison of cell cycle distribution (top, bottom left) and total cell number (bottom right) under same conditions as Figure 8 in CCP-PBM-PD (change in $\sigma \%$ and $\tau$ according to same steady state) and CCP-DDE-PD (black dashed line) under equivalent growth kinetics.

\section{Table captions}

Table 1: Model parameters and experimental methods needed to obtain them (the model source is clearly stated, otherwise all models use that specific parameter).

Table 2: Phase time parameters estimated in each of the three models as compared to the ones obtained experimentally.

Table 3: Residual sum of squares (RSS) for the parameter estimation in each model, for each cell line.

Table 4: Cell cycle times converted from CCP-DDE to CCP-PBM and CCP-ODE for the steady state conditions chosen $\left(G_{S S, \%}=79.3 \%, S_{S S \%}=16.0 \%, M_{S S \%}=4.8 \%\right.$, $\left.\alpha=\mu=\gamma=0.0157 h^{-1}\right)$. 